\title{
The stability for the Cauchy problem for elliptic equations*
}

\author{
Giovanni Alessandrini, ${ }^{\dagger}$ Luca Rondi, ${ }^{\ddagger}$ Edi Rosset, ${ }^{\S}$ and Sergio Vessellaף
}

\begin{abstract}
We discuss the ill-posed Cauchy problem for elliptic equations, which is pervasive in inverse boundary value problems modeled by elliptic equations.

We provide essentially optimal stability results, in wide generality and under substantially minimal assumptions.

As a general scheme in our arguments, we show that all such stability results can be derived by the use of a single building brick, the three-spheres inequality.
\end{abstract}

Mathematics Subject Classification (2000) Primary 35R25. Secondary 35B60, 35R30, 31A15, 30C62, 30G20.

Keywords Cauchy problem, elliptic equations, ill-posed problems, three-spheres inequalities, conditional stability, quasiconformal mappings

\section{Introduction}

Hadamard, in his paper of 1902 [37] where he laid the basis of the notion of well-posed problems, used the Cauchy problem for Laplace's equation as his first example of a problem which is not well-posed. Later, in 1923, he published [38] his well-known example of instability, see Subsection 1.1 below for further discussion and also Maz'ya and Shaposhnikova [70] for additional information.

It may be curious to note that in the same span of time, the physical relevance of this problem was to be encountered in the applications. For instance, in geophysical underground prospection, the geoelectrical method was initiated in those years, see for instance Stefanesco et al. [92] and the historical account in Zhdanov and Keller [100]. And in fact it is well acknowledged, by now, that the geoelectrical method involves, even in its most basic formulation, the solution of a Cauchy problem for Laplace's equation! On this respect, one can consult, for instance, the initial considerations in the book by Lavrent'ev, Romanov and Šišatskiı̌ [65].

\footnotetext{
${ }^{*}$ This work has been completed in spite of the indiscriminate budget cuts of the Italian Ministry of University and Research.

${ }^{\dagger}$ Dipartimento di Matematica e Informatica, Università degli Studi di Trieste, via Valerio 12/1, 34127 Trieste, Italy. E-mail: alessang@units.it

${ }^{\ddagger}$ Dipartimento di Matematica e Informatica, Università degli Studi di Trieste, via Valerio 12/1, 34127 Trieste, Italy. E-mail: rondi@units.it

${ }^{\S}$ Dipartimento di Matematica e Informatica, Università degli Studi di Trieste, via Valerio 12/1, 34127 Trieste, Italy. E-mail: rossedi@units.it

${ }^{\top}$ DIMAD, Università degli Studi di Firenze, via Lombroso 6/17, 50134 Firenze, Italy. E-mail: sergio.vessella@dmd.unifi.it
} 
Nowadays, it is widely recognized that the Cauchy problem for Laplace's equation, and more generally for elliptic equations, has a central position in all inverse boundary value problems which are modeled by means of elliptic partial differential equations, Inverse Scattering, Electrical Impedance Tomography, Optical Tomography, just to mention a few. The continuing interest on this kind of problem is documented by the number of publications which are currently appearing on this problem. For instance, we have recorded at least 15 papers explicitly devoted to this topic, which have appeared in the last three years on this Journal.

Therefore, we believe that it might be useful to formulate in a clear fashion the state of the art on the issue of stability, which is obviously a crucial cornerstone of the convergence analysis of any reconstruction procedure and also of the stability analysis of many nonlinear inverse boundary value problems, whose treatment involves, in one way or another, the analysis of an ill-posed Cauchy problem.

In this introduction we do not intend to present a complete discussion on the historical development on this subject since Hadamard, because various monographs, Lavrent'ev [64], Payne [83], Lavrent'ev, Romanov and Šišatskiǔ [65], Isakov [47, 48], Hörmander [43, 45], already contain abundant information on such development. However, besides stating and proving results of stability of sufficient generality and optimality it may be useful to discuss some different, although intertwined, lines of reasoning which, in our view, have led to the current state of the art.

\subsection{Instability and conditional stability}

In his essay of 1923 [38], Hadamard provided a fundamental example which shows that a solution of a Cauchy problem for Laplace's equation does not depend continuously upon the data. The example is as follows.

Consider the solution $u=u_{n}, n=1,2, \ldots$ to the Cauchy problem in the upper half plane

$$
\begin{cases}\Delta u=0, & \text { in }\left\{(x, y) \in \mathbb{R}^{2} \mid y>0\right\} \\ u(x, 0)=0, & \text { for every } x \in \mathbb{R} \\ u_{y}(x, 0)=A_{n} \sin n x, & \text { for every } x \in \mathbb{R}\end{cases}
$$

We have

$$
u_{n}=\frac{A_{n}}{n} \sin n x \sinh n y .
$$

If we choose $A_{n}=\frac{1}{n}$ or $A_{n}=\frac{1}{n^{p}}$ for some $p>0$, or even $A_{n}=e^{-\sqrt{n}}$, it turns out that

$$
u_{n, y}(x, 0) \rightarrow 0 \text { uniformly as } n \rightarrow \infty
$$

whereas, for any $y>0$,

$$
u_{n}(x, y)=\frac{A_{n}}{n} \sin n x \sinh n y \text { blows up as } n \rightarrow \infty .
$$

As is well-known since Tikhonov [95] the modern notion of stability for ill-posed problems (also called conditional stability) consists of estimating the dependence upon the data of the unknown solution of the problem at hand, when an a-priori bound on the solution itself is available. 
It is a remarkable fact that Hadamard eventually acknowledged that continuous dependence can be restored in presence of an a-priori bound. In fact, in his treatise of 1964 [39, p. 146] he wrote

"D'après un remarquable résultat dû a M. Pucci, l'absence de continuité de la solution $u$ du probléme de Cauchy considérée comme functionelle des données initiales est solidaire du fait que cette solution est susceptible d'augmenter infinitement: M. Pucci constate que le choses changent si l'on connait une borne supériore de le valeur absolute $|u| \cdot{ }^{\prime *}$

In fact, Hadamard is referring to a paper by Pucci [88] of 1955 where one of the first results of stability for the Cauchy problem for Laplace's equation was obtained. Let us recall that in the same years other stability estimates were obtained by John [52], Landis [60] and Lavrent'ev [62,63], see also Pucci [89] and John [53].

It may be instructing to observe that the same example by Hadamard may be used to exhibit the best possible rates of continuous dependence in presence of an a-priori bound. To this purpose let us describe a Cauchy problem in the most simple and favourable setting. We express the a-priori bound and the bounds on the data with respect to norms which can be considered as the natural ones in the standard variational formulation of Laplace's equation, but it will be evident that analogous results would be obtained also if other (reasonable) functional frameworks are considered.

Consider a Cauchy problem in a rectangle

$$
\begin{cases}\Delta u=0, & \text { in }(0, \pi) \times(0,1), \\ u(x, 0)=0, & \text { for every } x \in(0, \pi), \\ u_{y}(x, 0)=\psi(x), & \text { for every } x \in(0, \pi),\end{cases}
$$

and, in order to make things even simpler, we further assume a zero Dirichlet condition on the vertical sides of the rectangle

$$
u(0, y)=u(\pi, y)=0, \quad \text { for every } y \in(0,1)
$$

The natural function space of the solution $u$, in a variational setting, is the Sobolev space $H^{1}((0, \pi) \times(0,1))$ and thus, as a-priori information on the unknown solution $u$, we assume the bound on the Dirichlet integral

$$
\iint_{(0, \pi) \times(0,1)}\left(u_{x}^{2}+u_{y}^{2}\right) d x d y \leq E^{2}
$$

for a given $E>0$.

The prescribed inhomogeneous data $\psi$, which express the (partial) Neumann data on the lower horizontal side of the rectangle, naturally lives in the trace space $H^{-\frac{1}{2}}(0, \pi)$. Let us assume then that the following error bound is known

$$
\|\psi\|_{H^{-\frac{1}{2}}(0, \pi)} \leq \eta
$$

\footnotetext{
* "After a remarkable result due to Mr. Pucci, the lack of continuity of the solution $u$ of the Cauchy
} problem considered as a functional of initial data is joint to the fact that this solution is susceptible of infinite growth: Mr. Pucci observes that things change if one knows an upper bound of the absolute value $|u| . "$ 
for some given $\eta>0$.

A stability estimate would consist of a bound of some norm of $u$ evaluated inside the rectangle in terms of some function $\omega(\eta, E)$ which should be infinitesimal as $\eta \rightarrow 0^{+}$. The Hadamard example provides us with limitations on such infinitesimal rate. Let us choose once more

$$
\psi_{n}(x)=A_{n} \sin n x, n=1,2, \ldots
$$

and let us select $A_{n}$ in such a way that equality holds in (1.3). We obtain

$$
A_{n}^{2}=\frac{2}{\pi} \frac{2 n}{\sinh 2 n} E^{2} .
$$

Consequently, in (1.4) we have equality when $\eta=\eta_{n}$ where $\eta_{n}$ is given by

$$
\eta_{n}^{2}=E^{2} \frac{2}{\sinh 2 n} \sim 4 E^{2} e^{-2 n}, \text { as } n \rightarrow \infty .
$$

If we wish to estimate the $L^{2}$-norm of $u$ in the rectangle $(0, \pi) \times(0, T)$, for some $T \in(0,1]$, then we see that the solution to (1.1), (1.2) with $\psi$ given by (1.5), (1.6), satisfies

$$
\left\|u_{n}\right\|_{L^{2}((0, \pi) \times(0, T))}^{2}=\frac{E^{2}}{n \sinh 2 n}\left(\frac{\sinh 2 n T}{2 n}-1\right) \sim E^{2} \frac{e^{2 n(T-1)}}{2 n^{2}}, \text { as } n \rightarrow \infty .
$$

That is

$$
\left\|u_{n}\right\|_{L^{2}((0, \pi) \times(0, T))} \sim \frac{E}{\sqrt{2}}\left(\frac{\eta_{n}}{2 E}\right)^{(1-T)}\left(\log \frac{2 E}{\eta_{n}}\right)^{-1}, \text { as } n \rightarrow \infty .
$$

Therefore, if $T<1$, then the stability of the determination of $u$ up to the level $y=T$ is at best of Hölder type. Whereas, if we want to recover $u$ in all of its domain of definition (up to the top side of the rectangle, where no boundary data is prescribed) then the best possible rate of stability is logarithmic.

It is generally acknowledged that this phenomenon has a quite general character when dealing with the Cauchy problem for elliptic equations. And in fact the results in the following sections agree with such a scheme.

We shall distinguish between two types of results.

Stability estimates in the interior. A solution $u$ of a Cauchy problem is a-priori known to be bounded (with respect to some norm) on a connected open set $\Omega$, Cauchy data are prescribed on some portion $\Sigma$ of $\partial \Omega$ and we wish to estimate $u$ on some connected open subset $G$ of $\Omega$ which is at a positive distance from $\partial \Omega \backslash \Sigma$, the part of the boundary where no data are prescribed.

Global stability estimates. We want to estimate $u$ in some norm in all of $\Omega$ when an upper bound with a slightly stronger norm in the same set $\Omega$ is a-priori known, and, as before, Cauchy data are prescribed on a portion $\Sigma$ of $\partial \Omega$.

We reiterate that the Hadamard example tells us that for a stability estimate in the interior we cannot expect anything better than a Hölder rate, whereas for global stability the optimal rate will be of logarithmic type at most. 
A rather general treatment of stability in the interior is due to Payne [82] (see also Payne [81], Trytten [96]). Global stability estimates in a wide generality, that is for general elliptic operators and general domains, have been known and used for quite a while, but probably statements and proofs are not easily available in the literature. In fact, a surge of interest on this topic occurred in the 90's in connection with nonlinear inverse boundary value problems with unknown boundaries and global stability estimates were described and used (Alessandrini [4], Alessandrini and Di Benedetto [6], Beretta and Vessella [16], Bukhgeim, Cheng and Yamamoto [20,21], Alessandrini, Beretta, Rosset and Vessella [5], Cheng, Hon and Yamamoto [26,27]) but, unfortunately, most of the times such estimates were not explicitly stated as independent results. As an exception, we mention Takeuchi and Yamamoto [94, Theorem 10].

\subsection{Analytic continuation}

In the special case of two space variables, the Cauchy problem for Laplace's equation is equivalent to the problem of continuation of a complex analytic function from values prescribed on an arc. This problem was treated with great ingenuity by Carleman in 1926. His theory is expounded in the essay of 1926 [24] and his ideas have had a great influence in the subsequent developments of the theory. A modern treatment of the connection between the Cauchy problem and the analytic continuation in two dimensions can be found in $[65, \mathrm{Ch} .1$, $\S 2]$. The crucial tool for stability is the so-called method of harmonic measure. In fact, the seminal idea of this method can be traced back to Carleman [23], but a general formulation of this approach can be attributed to F. and R. Nevanlinna [79], see in this respect Goluzin [35, Ch. VII, § 4].

The two-dimensional theory for Cauchy problems maintains a special position also when dealing with elliptic operators with variable coefficients. Indeed, still with the aid of complex analytic methods, uniqueness, Alessandrini and Magnanini [8], and stability, Alessandrini and Rondi [10], can be obtained with no need of regularity assumptions on the coefficients (contrary to what happens in higher dimensions, see in this respect Subsection 1.3 below). An extended version of the harmonic measure technique has been developed also for the variable coefficients case in Alessandrini and Rondi [10]. In Section 3 below we shall discuss in more detail this kind of results.

\subsection{The Cauchy problem and the unique continuation property}

An issue which is strictly related to the Cauchy problem is the one of unique continuation. An elliptic operator $\mathcal{L}$ is said to have the (weak) unique continuation property if for any solution $u$ to $\mathcal{L} u=0$ in a connected open set $\Omega \subset \mathbb{R}^{n}$, that vanishes on an open subset $G \subset \Omega$, it follows $u \equiv 0$ in $\Omega$. A general proof of the equivalence of the uniqueness of the Cauchy problem with the weak unique continuation property can be traced back to Nirenberg [80]. This equivalence has been especially important in establishing the limits of validity of uniqueness in terms of the regularity of the coefficients of the elliptic operator involved. In fact it was shown by Pliš [86] that for an elliptic operator $\mathcal{L}$ in dimension $n \geq 3$, the unique continuation may fail if the coefficients of the principal part are Hölder continuous of any exponent smaller than 1. Further examples were obtained by Miller [71], see also Miller [72] for improvements and discussion on such counterexamples, and Mandache [69], Filonov [31] for further developments. 
The progress on this issue of unique continuation was initiated by Carleman [25], subsequent advances were due to Müller [76], Heinz [42], Hartman and Wintner [40], Cordes [28], Aronszajn [14]. Eventually, it was proved by Aronszajn, Krzywicki and Szarski [15] that the unique continuation property holds true when the coefficients in the principal part are Lipschitz continuous. Soon afterwards, Pliš produced the already mentioned example that shows that such Lipschitz continuity provides indeed the crucial threshold. A great deal of investigation followed, especially with the purpose of extending the unique continuation property to Schrödinger operators with singular potentials. With no ambition of completeness, let us mention Hörmander [44], Jerison and Kenig [50], Garofalo and Lin [32, 33], Fabes, Garofalo and Lin [30] and, more recently, Koch and Tataru [55].

\subsection{Three-spheres inequalities}

The unique continuation property is also connected to the problem of the stability for the Cauchy problem. In fact, proofs of the unique continuation property depend on inequalities which can also be applied to the estimation of stability. There are two families of such inequalities

- Carleman estimates,

- Three-spheres inequalities.

Both types have been succesfully used in the study of stability, and they are strictly intertwined, in fact three-spheres inequalities can be deduced by Carleman estimates.

In this paper, a central theme that we intend to stress is that three-spheres inequalities can be used as a universal building brick to derive optimal stability estimates. Our guiding idea shall be to confine all the hard-analysis which is required to the derivation of a basic inequality in a simple geometrical setting (the three-spheres) and then use it iteratively to adapt to general geometrical configurations.

We find quite instructing to remark at this point that Hadamard, the same mathematician who first pointed out the ill character of the Cauchy problem, was the one who first provided a cure (actually, even before the illness was diagnosed!). In fact, Hadamard first stated in 1896 [36] a three-circles inequality, which in its simplest manifestation is as follows. Given a holomorphic function $f$ in the disk $\{z \in \mathbb{C}|| z \mid<R\}$ then the function

$$
\log r \rightarrow \log \left(\max _{|z|=r}|f(z)|\right), 0<r<R
$$

is convex. Quoting once more Hadamard [36, p. 94]

“...je désignerai par $\eta$ le logarithme du module maximum de la fonction sur le cercle de rayon $\varepsilon^{\xi}$ (où $\xi$ est un nombre réel quelconque). Le lieu du point $(\xi, \eta)$ est une courbe $C$ qui tourne toujours sa concavité vers le $\eta$ positifs; ... "独

\footnotetext{
$\dagger$ "... I shall denote with $\eta$ the logarithm of the maximum modulus of the function on the circle of radius $\varepsilon^{\xi}$ (where $\xi$ is any real number). The locus of the points $(\xi, \eta)$ is a curve $C$ that always bends its concavity towards the positive $\eta$ 's; ..."
} 
In other terms, if $0<r_{1}<r_{2}<r_{3}<R$, then

$$
\max _{|z|=r_{2}}|f(z)| \leq\left(\max _{|z|=r_{1}}|f(z)|\right)^{\alpha}\left(\max _{|z|=r_{3}}|f(z)|\right)^{1-\alpha},
$$

where $\alpha \in(0,1)$ is given by

$$
\alpha=\frac{\log \frac{r_{3}}{r_{2}}}{\log \frac{r_{3}}{r_{1}}} .
$$

This inequality had a great influence in the following development of complex analysis [29] but it had also a seminal character in the study of unique continuation for elliptic equations. A three-spheres inequality for elliptic operators whose principal part coefficients are $C^{2}$ was proved by Landis [61], and in fact his proof was based on Carleman's type estimates. Another proof obtained by a method of differential inequalities for integral norms, which took the name of logarithmic convexity, was obtained by Agmon [3]. In the 70's the general concept of logarithmic convexity had indeed a notable influence in the analysis of various ill-posed problems for partial differential equations. The proceedings book edited by Knops [54] documents the advances in this direction.

With more precise connection with the stability for the Cauchy problem, this approach reached its apex in the work by Payne [81,82], see also the contribution by Trytten [96]. In particular in [82], Payne obtained stability estimates in the interior of Hölder type when the coefficients of the principal part are $C^{1}$.

Returning however to the more specific issue of the three-spheres inequalities, we mention that recent proofs have been obtained by Brummelhuis [19], and by Kukavica [58]. Both authors use, with some variations, the so-called method of the frequency function by Garofalo and Lin [32]. In fact the frequency function method can be viewed as a further notable advance and clarification of the ideas of logarithmic convexity.

We shall formulate various versions of the three-spheres inequality and we shall provide a proof which is modeled, with few adjustements, on the one by Kukavica [58]. Let us remark however that the same inequality might be obtained also by means of Carleman estimates. We refer to Vessella [99] for a general discussion of this approach, in the wider context of parabolic equations. Let us also quote Lin, Nakamura and Wang [67] for a very recent investigation in this direction.

\subsection{Doubling inequalities}

It cannot be omitted at this point that reasearch on unique continuation has been especially concentrated on the aspect of the strong unique continuation property, that is, if $u$ solves the elliptic equation $\mathcal{L} u=0$ in a connected open set $\Omega$ and $u$ vanishes of infinite order at

one point $x_{0} \in \Omega\left(\right.$ that is $u(x)=O\left(\left|x-x_{0}\right|^{N}\right)$ as $x \rightarrow x_{0}$, for every $\left.N=1,2, \ldots\right)$ then $u$ has to be zero everywhere.

This property does not have a direct connection with the stability of the Cauchy problem. However, quantitative versions of the strong unique continuation property have shown to be very useful in the study of stability of certain inverse boundary value problems. Such quantitative estimates are in fact the doubling inequality by Garofalo and Lin [32] and the doubling inequality at the boundary, see Adolfsson, Escauriaza and Kenig [2], Adolfsson and Escauriaza [1], Kukavica and Nyström [59]. Applications to elliptic inverse boundary 
value problems occurred for instance in Alessandrini, Rosset and Seo [12], Alessandrini, Beretta, Rosset and Vessella [5]. We recall also that such quantitative estimates have been used in connection with the problem of estimates of continuation from measurable sets, which is very much related to the Cauchy problem as well. In this respect let us mention Nadirashvili [77,78], Vessella [97,98], and Malinnikova [68].

\subsection{The scheme of a stability proof}

Our general scheme of proof for the stability of a Cauchy problem will be as follows.

I) First we prove a three-spheres inequality. We shall need an inequality of this kind not only for solutions of homogeneous elliptic equations but more generally for solutions of inhomogeneous equations $\mathcal{L} u=\mathcal{F}$ with an $H^{-1}$ right-hand side $\mathcal{F}$ (see Theorem 1.10).

II) Next we use iteratively the three-spheres inequality to obtain estimates of propagation of smallness. We assume an a-priori bound on a solution $u$ on its domain $\Omega$ and that $u$ is small in a given (small) ball $B_{r_{0}}\left(x_{0}\right) \subset \Omega$ and we estimate how small is $u$ in some larger connected open set $G \subset \Omega$. If $G$ is at a positive distance from $\partial \Omega$ we shall speak of estimates of propagation of smallness in the interior (see Theorem [5.1), instead if $G$ agrees with $\Omega$ we shall speak of global estimates of propagation of smallness (see Theorem 5.3 and also Remark 7.2 .

III) Then, given a solution of a Cauchy problem in $\Omega$ with data on a portion $\Sigma$ of $\partial \Omega$, we extend $u$ to an open set $\mathcal{A}$ outside of $\Omega$, whose boundary agrees with $\partial \Omega$ on a subset of $\Sigma$. We perform such extension in such a way that the extended function $\widetilde{u}$ solves in $\mathcal{A}$ an inhomogeneous equation with a right-hand side which is controlled in a Lipschitz fashion by the Cauchy data on $\Sigma$ (see Theorem 6.2 and the following Remark 6.3).

IV) Finally, we apply the estimates of propagation of smallness in the augmented domain $\widetilde{\Omega}=\overline{\Omega \cup \mathcal{A}}$ thus obtaining an interior stability estimate for the Cauchy problem in Theorem 1.7, and a global stability estimate in Theorem 1.9.

\subsection{The main hypotheses and statements}

In order to discuss in more detail the hypotheses that are used in our treatment it is necessary to introduce some notation and definitions.

Given $x \in \mathbb{R}^{n}$, we shall denote $x=\left(x^{\prime}, x_{n}\right)$, where $x^{\prime}=\left(x_{1}, \ldots, x_{n-1}\right) \in \mathbb{R}^{n-1}, x_{n} \in \mathbb{R}$. Given $x \in \mathbb{R}^{n}, r>0$, we shall use the following notation for balls and cylinders.

$$
\begin{gathered}
B_{r}(x)=\left\{y \in \mathbb{R}^{n}|| y-x \mid<r\right\}, \quad B_{r}=B_{r}(0), \\
B_{r}^{\prime}\left(x^{\prime}\right)=\left\{y^{\prime} \in \mathbb{R}^{n-1}|| y^{\prime}-x^{\prime} \mid<r\right\}, \quad B_{r}^{\prime}=B_{r}^{\prime}(0), \\
\Gamma_{a, b}(x)=\left\{y=\left(y^{\prime}, y_{n}\right) \in \mathbb{R}^{n}|| y^{\prime}-x^{\prime}|<a,| y_{n}-x_{n} \mid<b\right\}, \quad \Gamma_{a, b}=\Gamma_{a, b}(0) .
\end{gathered}
$$

Throughout this paper we shall denote by $\Omega$ a bounded open connected subset of $\mathbb{R}^{n}$. In places we shall assume that the boundary of $\Omega$ is Lipschitz according to the following definition. 
Definition 1.1 (Lipschitz regularity) We say that the boundary of $\Omega$ is of Lipschitz class with constants $\rho_{0}, M_{0}>0$, if, for any $P \in \partial \Omega$, there exists a rigid transformation of coordinates under which $P=0$ and

$$
\Omega \cap \Gamma_{\frac{\rho_{0}}{M_{0}}, \rho_{0}}(P)=\left\{x=\left(x^{\prime}, x_{n}\right) \in \Gamma_{\frac{\rho_{0}}{M_{0}}, \rho_{0}} \quad \mid \quad x_{n}>Z\left(x^{\prime}\right)\right\},
$$

where $Z: B_{\frac{\rho_{0}}{M_{0}}}^{\prime} \rightarrow \mathbb{R}$ is a Lipschitz function satisfying

$$
\begin{gathered}
Z(0)=0, \\
\left.\|Z\|_{C^{0,1}\left(B^{\prime} \rho_{0}\right.}^{M_{0}}\right) \\
\leq M_{0} \rho_{0} .
\end{gathered}
$$

Remark 1.2 For practical purposes it will turn out useful to assume throughout that $M_{0} \geq 1$. In fact it is evident from Definition 1.1 that conditions (1.10)-(1.12) continue to hold if $M_{0}$ is increased.

Remark 1.3 Throughout this paper we shall use the convention to normalize all norms in such a way that they are dimensionally equivalent to their argument and coincide with the standard definition when the dimensional parameter $\rho_{0}$ equals 1 . For instance, the norm appearing above is meant as follows

$$
\left.\|Z\|_{C^{0,1}\left(B_{\frac{\rho_{0}}{M_{0}}}^{\prime}\right)}=\|Z\|_{L^{\infty}\left(B_{\frac{\rho_{0}}{M_{0}}}^{\prime}\right)}{ }^{+\rho_{0}\|\nabla Z\|}{ }_{L^{\infty}\left(B_{B_{0}^{\prime}}^{\prime}\right.}{ }^{\rho_{0}}\right) .
$$

Similarly, we shall set

$$
\begin{gathered}
\|u\|_{L^{2}(\Omega)}=\rho_{0}^{-\frac{n}{2}}\left(\int_{\Omega} u^{2}\right)^{\frac{1}{2}} \\
\|u\|_{H^{1}(\Omega)}=\rho_{0}^{-\frac{n}{2}}\left(\int_{\Omega} u^{2}+\rho_{0}^{2} \int_{\Omega}|\nabla u|^{2}\right)^{\frac{1}{2}}
\end{gathered}
$$

and so on for boundary and trace norms such as $\|\cdot\|_{L^{2}(\partial \Omega)},\|\cdot\|_{H^{\frac{1}{2}(\partial \Omega)}},\|\cdot\|_{H^{-\frac{1}{2}}(\partial \Omega)}$.

In some instances we shall require that only a limited open portion $\Sigma$ of $\partial \Omega$ be Lipschitz in the following sense. Some further notation is necessary. We shall denote

$$
\Sigma^{\prime}=\partial \Omega \backslash \Sigma,
$$

and, for every $P \in \Sigma$, we set

$$
\begin{gathered}
r(P)=\operatorname{dist}\left(P, \Sigma^{\prime}\right), \\
\rho(P)=\min \left\{\rho_{0}, \frac{r(P) M_{0}}{\sqrt{1+M_{0}^{2}}}\right\} .
\end{gathered}
$$


Definition 1.4 An open subset $\Sigma \subset \partial \Omega$ is said to be an open Lipschitz portion of $\partial \Omega$ with constants $\rho_{0}, M_{0}>0$, if, for any $P \in \Sigma$, there exists a rigid transformation of coordinates under which $P=0$ and

$$
\Omega \cap \Gamma_{\frac{\rho(P)}{M_{0}}, \rho(P)}(P)=\left\{x=\left(x^{\prime}, x_{n}\right) \in \Gamma_{\frac{\rho(P)}{M_{0}}, \rho(P)} \quad \mid \quad x_{n}>Z\left(x^{\prime}\right)\right\},
$$

where $Z: B_{\frac{\rho_{0}}{M_{0}}}^{\prime} \rightarrow \mathbb{R}$ is a Lipschitz function satisfying (1.11)-(1.12).

We shall also need an assumption on $\Sigma$ in order to control from below its smallness.

Definition 1.5 We shall say that $\Sigma$ has size at least $\rho_{1}, 0<\rho_{1} \leq \rho_{0}$, if there exists at least one point $P \in \Sigma$ such that

$$
\rho(P) \geq \rho_{1} .
$$

The elliptic operators that we shall consider are of the following form

$$
\mathcal{L} u=\operatorname{div}(A \nabla u)+c u
$$

where $A=A(x)=\left\{a_{i j}(x)\right\}, x \in \mathbb{R}^{n}$, is a real-valued symmetric $n \times n$ matrix such that its entries are measurable and it satisfies, for a given constant $K \geq 1$, the ellipticity condition

$$
K^{-1}|\xi|^{2} \leq A(x) \xi \cdot \xi \leq K|\xi|^{2}, \quad \text { for almost every } x \in \mathbb{R}^{n} \text {, for every } \xi \in \mathbb{R}^{n} .
$$

Furthermore, when $n \geq 3$, we also assume that, for a given constant $L>0$, the following Lipschitz continuity holds

$$
|A(x)-A(y)| \leq \frac{L}{\rho_{0}}|x-y|, \quad \text { for every } x, y \in \mathbb{R}^{n} .
$$

Concerning the zero order term, we assume that $c \in L^{\infty}\left(\mathbb{R}^{n}\right)$ with

$$
\|c\|_{L^{\infty}\left(\mathbb{R}^{n}\right)} \leq \frac{\kappa}{\rho_{0}^{2}}
$$

Here and for the rest of this paper we shall assume that $A$ is a symmetric matrix of coefficients satisfying the ellipticity condition (1.19) and such that, if $n \geq 3$, it also satisfies the Lipschitz condition (1.20). We emphasize that, in all the following statements, whenever a constant is said to depend on $L$ (among other quantities) it is understood that such dependence occurs only when $n \geq 3$.

We remark once and for all that also first order terms with bounded coefficients could be added to the treatment with little additional effort. We have chosen to confine ourselves to the above variational structure (1.18) because we believe that (1.18) provides a sufficiently wide and useful setting for enough applications. We insist however that we allow the presence of the zero order term, with no sign, nor smallness, condition on the coefficient $c$ because of the importance of the applications to wave phenomena at a fixed wavenumber.

Let us now introduce the rigorous weak formulation of the Cauchy problem that we shall use. First it is necessary to introduce some further notation and some function spaces.

The solution $u$ will be assumed to belong to the $H^{1}(\Omega)$ space and the following a-priori bound will be prescribed

$$
\|u\|_{H^{1}(\Omega)} \leq E,
$$


for some given $E>0$, for the purpose of a global stability estimate, whereas for the stability in the interior we shall more simply require that, given $E_{0}>0$,

$$
\|u\|_{L^{2}(\Omega)} \leq E_{0}
$$

We shall fix $\Sigma$ as an open connected portion of $\partial \Omega$ and we shall always assume it to be Lipschitz.

We shall consider as test functions space the space $H_{c o}^{1}(\Omega \cup \Sigma)$ consisting of the functions $\varphi \in H^{1}(\Omega)$ having support compactly contained in $\Omega \cup \Sigma$. We denote by $H_{c o}^{\frac{1}{2}}(\Sigma)$ the class of $H^{\frac{1}{2}}(\Sigma)$ traces of functions $\varphi \in H_{c o}^{1}(\Omega \cup \Sigma)$. We then define $H^{-\frac{1}{2}}(\Sigma)$ as the dual space to $H_{c o}^{\frac{1}{2}}(\Sigma)$ based on the $L^{2}(\Sigma)$ dual pairing.

The Cauchy data $g, \psi$ will be taken in their natural trace spaces, namely

$$
g \in H^{\frac{1}{2}}(\Sigma), \psi \in H^{-\frac{1}{2}}(\Sigma) .
$$

As bounds on the Cauchy data we require

$$
\|g\|_{H^{\frac{1}{2}(\Sigma)}}+\rho_{0}\|\psi\|_{H^{-\frac{1}{2}(\Sigma)}} \leq \eta
$$

To begin with, let us consider the following more or less standard formulation of a Cauchy problem

$$
\begin{cases}\operatorname{div}(A \nabla u)+c u=f, & \text { in } \Omega, \\ u=g, & \text { on } \Sigma, \\ A \nabla u \cdot \nu=\psi, & \text { on } \Sigma,\end{cases}
$$

where the right-hand side $f$ can be assumed in $L^{2}\left(\mathbb{R}^{n}\right)$.

The corresponding rigorous weak formulation of the Cauchy problem would be to find $u \in H^{1}(\Omega)$ such that $u_{\mid \Sigma}=g$ in the trace sense and

$$
\int_{\Omega}(A \nabla u \cdot \nabla \varphi-c u \varphi)=\int_{\Sigma} \psi \varphi-\int_{\Omega} f \varphi, \quad \text { for every } \varphi \in H_{c o}^{1}(\Omega \cup \Sigma) .
$$

Here, the integral $\int_{\Sigma} \psi \varphi$ is to be properly interpreted as the dual pairing between $H^{-\frac{1}{2}}(\Sigma)$ and $H_{c o}^{\frac{1}{2}}(\Sigma)$. Note that the right hand side in (1.27) represents a bounded linear functional over $H_{c o}^{1}(\Omega \cup \Sigma)$. An even more general bounded functional over $H_{c o}^{1}(\Omega \cup \Sigma)$ could be written as follows

$$
\mathcal{F}(\varphi)=\int_{\Sigma} \psi \varphi-\int_{\Omega}(f \varphi-F \cdot \nabla \varphi), \quad \text { for every } \varphi \in H_{c o}^{1}(\Omega \cup \Sigma)
$$

where $F \in L^{2}\left(\mathbb{R}^{n} ; \mathbb{R}^{n}\right)$ is a given vector field with $L^{2}$ components. It will be convenient for us to admit such type of right-hand side in our formulation. Observe, however, that such a representation of members of $\left(H_{c o}^{1}(\Omega \cup \Sigma)\right)^{*}$ is exceedingly non-unique (and indeed we shall fruitfully take advantage of this non-uniqueness in Theorem 6.2).

Hence we shall prescribe

$$
\|f\|_{L^{2}\left(\mathbb{R}^{n}\right)}+\frac{1}{\rho_{0}}\|F\|_{L^{2}\left(\mathbb{R}^{n} ; \mathbb{R}^{n}\right)} \leq \frac{\varepsilon}{\rho_{0}^{2}},
$$

for a given $\varepsilon>0$ and we shall formulate the rigorous weak formulation of the Cauchy problem as follows. 
Problem 1.6 (The weak formulation of the Cauchy problem) To find $u \in H^{1}(\Omega)$ such that $u_{\mid \Sigma}=g$ in the trace sense and

$$
\int_{\Omega}(A \nabla u \cdot \nabla \varphi-c u \varphi)=\int_{\Sigma} \psi \varphi-\int_{\Omega}(f \varphi-F \cdot \nabla \varphi), \quad \text { for every } \varphi \in H_{c o}^{1}(\Omega \cup \Sigma) .
$$

We remark that an abstract interpretation of the above stated problem could be expressed as follows

$$
\begin{cases}\operatorname{div}(A \nabla u)+c u=\mathcal{F}, & \text { in }\left(H_{c o}^{1}(\Omega \cup \Sigma)\right)^{*}, \\ u_{\mid \Sigma}=g, & \text { in } H^{\frac{1}{2}}(\Sigma),\end{cases}
$$

where $\mathcal{F}$ is given by (1.28). Unfortunately, this abstract formulation hides within its first equation a boundary contribution which should express the Neumann condition on $\Sigma$. Roughly speaking, such boundary contribution can be detected, if one formally integrates by parts the terms involving $\nabla \varphi$ appearing in (1.30), and formally obtains

$$
\begin{cases}\operatorname{div}(A \nabla u)+c u=f+\operatorname{div} F, & \text { in } \Omega, \\ u=g, & \text { on } \Sigma, \\ A \nabla u \cdot \nu=\psi+F \cdot \nu, & \text { on } \Sigma,\end{cases}
$$

and it is evident that this formal expression incorporates as a special case (1.26). We reiterate, however, that the true interpretation of the Cauchy problem that we shall use is given by Problem 1.6, but at the same time we emphasize that the full strength of such a rigorous formulation shall be used at a single step in our arguments only, and specifically in the above mentioned Theorem 6.2. In all remaining estimates occurring in this paper we shall merely make use of the interior weak formulation of the elliptic equation

$$
\operatorname{div}(A \nabla u)+c u=f+\operatorname{div} F
$$

which can be viewed in the more customary sense of $H^{-1}(\Omega)=\left(H_{0}^{1}(\Omega)\right)^{*}$.

Our main stability estimates are contained in the following theorems.

Theorem 1.7 (Stability in the interior for the Cauchy problem) Let $u \in H^{1}(\Omega)$ be a weak solution to the Cauchy Problem 1.6, where $\Sigma$ satisfies the conditions in Definition 1.4 and Definition 1.5, $f \in L^{2}\left(\mathbb{R}^{n}\right)$ and $F \in L^{2}\left(\mathbb{R}^{n} ; \mathbb{R}^{n}\right)$ satisfy (1.29) and $g \in H^{\frac{1}{2}}(\Sigma)$, $\psi \in H^{-\frac{1}{2}}(\Sigma)$ satisfy (1.25). There exists $\bar{h}, 0<\bar{h}<\frac{\rho_{1}}{8 M_{0}}$, with $\frac{\bar{h}}{\rho_{0}}$ only depending on $K, L$, $\kappa, M_{0}$ and $\frac{\rho_{0}}{\rho_{1}}$, such that, assuming the a-priori bound

$$
\|u\|_{L^{2}(\Omega)} \leq E_{0}
$$

then, for every $h, 0<h \leq \bar{h}$, and for every connected open set $G \subset \Omega$ such that

$$
\begin{gathered}
\operatorname{dist}(G, \partial \Omega) \geq h, \\
\operatorname{dist}(P, G)<\frac{\rho_{1}}{8 M_{0}},
\end{gathered}
$$


where $P \in \Sigma$ is the point appearing in Definition 1.5, we have

$$
\|u\|_{L^{2}(G)} \leq C(\varepsilon+\eta)^{\delta}\left(E_{0}+\varepsilon+\eta\right)^{1-\delta}
$$

where $C>0$ and $\delta \in(0,1)$ satisfy

$$
C=C_{1}\left(\frac{|\Omega|}{h^{n}}\right)^{\frac{1}{2}}
$$

and

$$
\delta \geq \alpha^{\frac{C_{2}|\Omega|}{h^{n}}}
$$

with $\alpha \in(0,1)$ only depending on $K, L$ and $\kappa, C_{1}$ only depending on $K, L, \kappa, M_{0}$ and $\frac{\rho_{0}}{\rho_{1}}$, and $C_{2}$ only depending on $K$.

Remark 1.8 Let us observe that stability with a Hölder rate could be obtained also when the connected subset $G$ of $\Omega$ is allowed to touch the boundary portion $\Sigma$, while remaining at a positive distance from its complement $\Sigma^{\prime}$. This fact will turn out to be evident from the proof, see also Remark 6.5. We have chosen the present formulation because in this way a more effective evaluation of the constants $C$ and $\delta$ is obtained in terms of the parameter $h$, which controls the distance of $G$ from the whole boundary $\partial \Omega$. It would also be possible to obtain analogous results in terms of the distance of $G$ from $\Sigma^{\prime}=\partial \Omega \backslash \Sigma$ at the price of assuming more information on the shape and regularity of the boundary of $\Sigma$ within $\partial \Omega$.

Theorem 1.9 (Global stability for the Cauchy problem) Let $\Omega$ be a connected open set of Lipschitz class according to Definition 1.1. Let $u \in H^{1}(\Omega)$ be a weak solution to the Cauchy Problem 1.6, where $f \in L^{2}\left(\mathbb{R}^{n}\right)$ and $F \in L^{2}\left(\mathbb{R}^{n} ; \mathbb{R}^{n}\right)$ satisfy $(\underline{1.29})$ and $g \in H^{\frac{1}{2}}(\Sigma)$, $\psi \in H^{-\frac{1}{2}}(\Sigma)$ satisfy (1.25). If u satisfies the a-priori assumption

$$
\|u\|_{H^{1}(\Omega)} \leq E
$$

then

$$
\|u\|_{L^{2}(\Omega)} \leq(E+\varepsilon+\eta) \omega\left(\frac{\varepsilon+\eta}{E+\varepsilon+\eta}\right)
$$

where

$$
\omega(t) \leq \frac{C}{\left(\log \frac{1}{t}\right)^{\mu}}, \quad \text { for } t<1 .
$$

where $C>0$ and $\mu, 0<\mu<1$, only depend on $K, L, \kappa, M_{0}, \frac{\rho_{0}}{\rho_{1}}$ and $\frac{|\Omega|}{\rho_{0}^{n}}$.

According to the scheme already illustrated in Subsection [1.6 our first step will be the proof of a three-spheres inequality. In fact our basic building brick will be the following

Theorem 1.10 (Three-spheres inequality) Let $u \in H^{1}\left(B_{R}\right)$ be a weak solution to the inhomogeneous elliptic equation

$$
\operatorname{div}(A \nabla u)+c u=f+\operatorname{div} F, \quad \text { in } B_{R},
$$


where $f \in L^{2}\left(\mathbb{R}^{n}\right)$ and $F \in L^{2}\left(\mathbb{R}^{n} ; \mathbb{R}^{n}\right)$ satisfy (1.29). Then, for every $r_{1}, r_{2}, r_{3}$, with $0<r_{1}<r_{2}<r_{3} \leq R$,

$$
\|u\|_{L^{2}\left(B_{r_{2}}\right)} \leq C\left(\|u\|_{L^{2}\left(B_{r_{1}}\right)}+\varepsilon\right)^{\alpha}\left(\|u\|_{L^{2}\left(B_{r_{3}}\right)}+\varepsilon\right)^{1-\alpha}
$$

where $C>0$ and $\alpha, 0<\alpha<1$, only depend on $K, L, \kappa, \max \left\{\frac{R}{\rho_{0}}, 1\right\}, \frac{r_{2}}{r_{1}}$ and $\frac{r_{3}}{r_{2}}$.

Our strategy for the proof of such an inequality breaks down in the following steps.

a) First we prove a three-spheres inequality for the homogeneous equation in pure principal part (Theorem 2.1) with some limitations on the radii, namely $0<r_{1}<r_{2}<r_{3} / K \leq$ $r_{3} \leq R$, when $n \geq 3$. In two dimensions, a three-spheres (in fact circles) inequality may be obtained, with a different technique, without the limitations on the radii and with a possibly discontinuous coefficient matrix $A$ (Theorem 3.11).

b) Next we adapt the proof to operators also containing the zero order term (Theorem 4.1).

c) We include the presence of the right-hand side in the equation (Theorem 4.4).

d) We remove the limitations on the radii, finally obtaining the above stated Theorem 1.10 .

We shall also consider estimates of propagation of smallness, in Section 5. The main results will be Theorem 5.1 (propagation in the interior) and Theorem 5.3 (global propagation). In fact, Theorem 5.1 is obtained as a consequence of the three-spheres inequality with restrictions on the radii, Theorem 4.4, and the above mentioned step d) is obtained as a special case of Theorem 5.1 .

In Section 6, we prove the extension argument (Theorem 6.2) which enables to apply the estimates of propagation of smallness (Theorems 5.1 and 5.3) to complete the proofs of Theorems 1.7 and 1.9 .

Finally, we shall address the problem of global stability when no regularity assumption is available on the domain $\Omega$. This situation in fact often occurs in inverse problems with unknown boundaries $[4,5,10,22,73,74,91,99]$. If two solutions of the same equation have different domains of definition, then their difference solves an equation in the intersection of the two domains. Such an intersection may be, in principle, highly nonsmooth, even if the starting domains satisfy some a-priori regularity assumptions. It is then useful to derive a preliminary, maybe very weak, global stability for such difference of solutions in their common domain of definition (or at least in one of its connected components). We believe that the present formalization of this argument, expressed in Theorem 7.1, might be a useful tool for future use in other inverse problems with unknown boundaries. Of course some assumptions will be needed anyhow. The basic one that we shall use on $\Omega$ is rather weak and can be summarized as follows. We shall assume that there exists a family $\left\{G_{h}\right\}$ of connected subsets invading $\Omega$ such that the measure of the difference $\left|\Omega \backslash G_{h}\right|$ is controlled by a given power $h^{\vartheta}$ of the distance $h$ of $G_{h}$ from $\partial \Omega$. It may be easy to predict that, with such weak hypotheses, only a weak result can be obtained. In fact we are able to achieve only a stability of $\log$-log-type. 


\subsection{Concluding remarks}

We believe that the above theorems provide optimal results of stability for the Cauchy problem. The optimality is achieved in many respects.

1) The regularity assumptions on the coefficients of the principal part are minimal, Lipschitz continuity when $n \geq 3$ and $L^{\infty}$ when $n=2$. In fact these are known to be optimal conditions for uniqueness.

2) The regularity assumptions on the boundary are kept to a minimum. In fact assuming Lipschitz regularity of the portion $\Sigma$ of $\partial \Omega$ where the Cauchy data are assigned seems to be a nearly minimal condition, just in order to give sense to the Cauchy problem. On the other hand, when dealing with global stability, some conditions on the boundary, which may ensure the uniform reachability of the boundary points from the interior, seem necessary and Lipschitz regularity appears to be enough general and at the same time meaningful in terms of applications. More general reachability conditions could be considered, such as the NTA corkscrew condition of Jerison and Kenig [51]. Let us mention in this direction the related investigation by Rondi [91].

3) The Cauchy data are evaluated in their natural spaces. This is indeed a slight improvement with respect to previous studies where typically $H^{1}(\Sigma)$-norm on $g$ and $L^{2}(\Sigma)$-norm on $\psi$ are considered, see Payne [82], Isakov [48], Takeuchi and Yamamoto [94].

4) As already discussed, the stability rates obtained have an optimal character. The quantities in the stability estimates which might require further investigation are the constants and the exponents (for instance $C$ and $\delta$ in Theorem 1.7 and $C$ and $\mu$ in Theorem 1.9). It would be interesting, although possibly rather difficult, to simultaneously optimize such pairs of quantities with respect to the geometry and also with respect to the coefficients of the equation. In connection to this issue we wish to mention some remarkable results by Hrycak and Isakov [46] and Subbarayappa and Isakov [93] who have considered the Helmholtz equation $\Delta u+k^{2} u=0$ and proved that the stability estimates improve as $|k|$ increases, in the sense that, as $|k| \rightarrow \infty$, the logarithmic term becomes negligible, whereas a Hölder term prevails. See also Isakov [49] for a related analysis with a more general equation.

The plan of the paper is as follows. Section 2 contains the proof of a three-spheres inequality for a homogeneous equation in pure principal part, the main result being Theorem 2.1. In Section 3 we investigate the two-dimensional case. We illustrate the connection with quasiconformal mappings in Proposition 3.1. We introduce the notion of $\mathcal{L}_{A}$-harmonic measure, Definition 3.5, and we apply it to stability estimates in the interior for Cauchy problems, Theorems 3.7 and 3.8. Finally, we deduce various forms of three-spheres (circles) inequalities, Theorem 3.10 and Theorem 3.11 which will be the one we shall use in the rest of the paper (together with Theorem 2.1). In Section 4 we adapt the previously obtained three-spheres inequalities in order to encompass equations containing the zero order term and an inhomogeneous right-hand side. The final result of this section is given in Theorem 4.4. Section 5 is devoted to estimates of propagation of smallness. In Theorem 5.1 we prove the interior estimate. As we already mentioned, as a corollary we also derive the proof of the above stated Theorem 1.10 . Next, in Theorem 5.3 we prove the global estimate. 
Section [6] contains the proof of the two main stability theorems for the Cauchy problem, Theorems 1.7 and 1.9. As we already illustrated, Section 7 deals with a global stability estimate for the Cauchy problem when no regularity is assumed on $\Omega$, Theorem 7.1 .

\section{The basic three-spheres inequality, arbitrary dimension}

Let us begin by considering the homogeneous elliptic equation in pure principal part

$$
\operatorname{div}(A \nabla u)=0, \quad \text { in } B_{R},
$$

where $A$ satisfies (1.19) and (1.20), for given constants $K \geq 1$ and $L>0$.

Theorem 2.1 (Three-spheres inequality - pure principal part) If the above stated hypotheses hold, for every $r_{1}, r_{2}, r_{3}$, with $0<r_{1}<r_{2}<\frac{r_{3}}{K} \leq r_{3} \leq R$,

$$
\|u\|_{L^{2}\left(B_{r_{2}}\right)} \leq Q\|u\|_{L^{2}\left(B_{r_{1}}\right)}^{\alpha}\|u\|_{L^{2}\left(B_{r_{3}}\right)}^{1-\alpha},
$$

where $Q \geq 1$ only depends on $K, L, \max \left\{\frac{R}{\rho_{0}}, 1\right\}$, and where

$$
\alpha=\frac{\log \frac{r_{3}}{K r_{2}}}{\log \frac{r_{3}}{K r_{2}}+C \log \frac{K r_{2}}{r_{1}}}
$$

with $C>0$ only depending on $K, L$ and $\max \left\{\frac{R}{\rho_{0}}, 1\right\}$.

Remark 2.2 It should be stressed here that, in the following sections, we shall not use the full strength of Theorem 2.1 with the essentially explicit formula (2.3) for the exponent of the three-spheres inequality. Actually, we shall merely use the fact that $\alpha$ only depends on $K, L, \max \left\{\frac{R}{\rho_{0}}, 1\right\}, \frac{r_{2}}{r_{1}}, \frac{r_{3}}{r_{2}}$. We have just recorded here the representation (2.3) because it may be of independent interest. We recall, in particular, that a three-spheres inequality (2.2) with exponent in the form (2.3) actually implies the strong unique continuation property in a rather straightforward fashion. The argument is as follows. We fix $r_{2}, r_{3}$ and we allow $r_{1} \rightarrow 0$, in this case

$$
\alpha=\alpha\left(r_{1}\right) \geq \frac{C_{1}}{\log \frac{C_{2}}{r_{1}}}
$$

With no loss of generality we may assume also $\|u\|_{L^{2}\left(B_{r_{3}}\right)} \leq 1$. If for every $N=1,2, \ldots$ we have $\|u\|_{L^{2}\left(B_{r}\right)}=O\left(r^{N}\right)$ as $r \rightarrow 0$, then, for every $N$, there exists $r^{\prime}>0$ such that

$$
\|u\|_{L^{2}\left(B_{r_{1}}\right)} \leq\left(\frac{r_{1}}{C_{2}}\right)^{N}, \text { for every } r_{1}<r^{\prime}
$$

consequently, by (2.2),

$$
\|u\|_{L^{2}\left(B_{r_{2}}\right)} \leq Q\left(\frac{r_{1}}{C_{2}}\right)^{\frac{N C_{1}}{\log \frac{C_{2}}{r_{1}}}}=Q e^{-C_{1} N} \rightarrow 0, \text { as } N \rightarrow \infty .
$$

See also Morassi, Rosset and Vessella [75] where this aspect was investigated in more depth. 
As a first step, we shall prove a three-spheres inequality under the following additional normalization hypothesis

$$
A(0)=I d
$$

Given a weak solution $u \in H^{1}\left(B_{R}\right)$ to equation (2.1), let us define

$$
\begin{aligned}
& \mu(x)=\frac{A(x) x \cdot x}{|x|^{2}}, \\
& H(r)=\int_{\partial B_{r}} \mu u^{2} .
\end{aligned}
$$

Theorem 2.3 (Three-spheres inequality - normalized case) If the previously stated hypotheses hold, for every $r_{1}, r_{2}, r_{3}$, with $0<r_{1}<r_{2}<r_{3} \leq R$,

$$
H\left(r_{2}\right) \leq Q H\left(r_{1}\right)^{\alpha} H\left(r_{3}\right)^{1-\alpha},
$$

where $Q \geq 1$ only depends on $K, L, \max \left\{\frac{R}{\rho_{0}}, 1\right\}$, and where

$$
\alpha=\frac{\log \frac{r_{3}}{r_{2}}}{\log \frac{r_{3}}{r_{2}}+C \log \frac{r_{2}}{r_{1}}},
$$

with $C>0$ only depending on $K, L$ and $\max \left\{\frac{R}{\rho_{0}}, 1\right\}$.

Remark 2.4 Since $K^{-1} \leq \mu \leq K$, we have

$$
\int_{\partial B_{r_{2}}} u^{2} \leq Q\left(\int_{\partial B_{r_{1}}} u^{2}\right)^{\alpha}\left(\int_{\partial B_{r_{3}}} u^{2}\right)^{1-\alpha}
$$

and, by integration and by Hölder inequality, we also obtain

$$
\int_{B_{r_{2}}} u^{2} \leq Q\left(\int_{B_{r_{1}}} u^{2}\right)^{\alpha}\left(\int_{B_{r_{3}}} u^{2}\right)^{1-\alpha} .
$$

Let us first derive Theorem 2.1 from Theorem 2.3.

Proof of Theorem 2.1 Let us introduce the change of variables

$$
y=J x,
$$

where $J=\sqrt{A^{-1}(0)}$ and let us consider, for any $r>0$, the ellipsoid

$$
\mathcal{E}_{r}=\left\{x \in \mathbb{R}^{n} \mid\left(A(0)^{-1} x\right) \cdot x<r^{2}\right\} .
$$

We have that $\mathcal{E}_{r}=J^{-1}\left(B_{r}\right)$ and

$$
B_{\frac{r}{\sqrt{K}}} \subset \mathcal{E}_{r} \subset B_{\sqrt{K} r}
$$


The function $v(y)=u\left(J^{-1} y\right)$ satisfies the elliptic equation

$$
\operatorname{div}(\widetilde{A} \nabla v)=0, \quad \text { in } B_{\frac{R}{\sqrt{K}}},
$$

where $\widetilde{A}(y)=J A\left(J^{-1} y\right) J$. It is straightforward to verify that

$$
\begin{gathered}
K^{-2}|\xi|^{2} \leq \widetilde{A}(y) \xi \cdot \xi \leq K^{2}|\xi|^{2}, \quad \text { for every } y \in \mathbb{R}^{n}, \xi \in \mathbb{R}^{n}, \\
\left|\widetilde{A}\left(y_{1}\right)-\widetilde{A}\left(y_{2}\right)\right| \leq K^{\frac{3}{2}} \frac{L}{\rho_{0}}\left|y_{1}-y_{2}\right|, \quad \text { for every } y_{1}, y_{2} \in \mathbb{R}^{n}, \\
\widetilde{A}(0)=I d .
\end{gathered}
$$

Therefore $\widetilde{A}$ satisfies the hypotheses of Theorem 2.3. Let $\rho_{1}=\frac{r_{1}}{\sqrt{K}}, \rho_{2}=\sqrt{K} r_{2}, \rho_{3}=\frac{r_{3}}{\sqrt{K}}$. Since $0<r_{1}<r_{2}<\frac{r_{3}}{K} \leq r_{3} \leq R$, we have that $0<\rho_{1}<\rho_{2}<\rho_{3} \leq \frac{R}{\sqrt{K}}$, and by (2.10) we have

$$
\int_{B_{\rho_{2}}} v^{2} \leq Q\left(\int_{B_{\rho_{1}}} v^{2}\right)^{\alpha}\left(\int_{B_{\rho_{3}}} v^{2}\right)^{1-\alpha},
$$

where $Q \geq 1$ only depends on $K, L, \max \left\{\frac{R}{\rho_{0}}, 1\right\}$, and where

$$
\alpha=\frac{\log \frac{\rho_{3}}{\rho_{2}}}{\log \frac{\rho_{3}}{\rho_{2}}+C \log \frac{\rho_{2}}{\rho_{1}}},
$$

with $C>0$ only depending on $K, L$ and $\max \left\{\frac{R}{\rho_{0}}, 1\right\}$. Coming back to the old variables we have

$$
\int_{\mathcal{E}_{\rho_{2}}} u^{2} \leq Q\left(\int_{\mathcal{E}_{\rho_{1}}} u^{2}\right)^{\alpha}\left(\int_{\mathcal{E}_{\rho_{3}}} u^{2}\right)^{1-\alpha},
$$

and recalling (2.13), the thesis follows.

The proof of Theorem 2.3 is essentially based on the following three lemmas.

Lemma 2.5 The function $H(r)$ is absolutely continuous in $(0, R)$ and, for almost every $r \in(0, R)$,

$$
H^{\prime}(r)=\frac{1}{r} \int_{\partial B_{r}}\left(-A \nu \cdot \nu+\operatorname{tr}(A)+r \partial_{x_{i}} a_{i j} \nu_{j}\right) u^{2}+2 \int_{\partial B_{r}} u A \nabla u \cdot \nu .
$$

Proof We have that

$$
\begin{aligned}
H(r)=\frac{1}{r} \int_{\partial B_{r}} u^{2} A(x) x \cdot \frac{x}{|x|} & =\frac{1}{r} \int_{\partial B_{r}} u^{2} A(x) x \cdot \nu= \\
& =\frac{1}{r} \int_{B_{r}} \operatorname{div}\left(u^{2} A(x) x\right)=\frac{1}{r} \int_{0}^{r} d s \int_{\partial B_{s}} \operatorname{div}\left(u^{2} A(x) x\right),
\end{aligned}
$$


where $\nu$ denotes the outer unit normal to $B_{r}$. Since, by well-known regularity results [34, Theorem 8.32], $u \in C_{l o c}^{1, \alpha}\left(B_{R}\right)$ and since $A \in C^{0,1}\left(B_{R}\right)$, we have that $\operatorname{div}\left(u^{2} A(x) x\right) \in$ $L^{\infty}\left(B_{R}\right)$ and therefore $H(r)$ is absolutely continuous in $(0, R)$. From (2.22), we have

$$
\begin{aligned}
H^{\prime}(r) & =-\frac{1}{r} H(r)+\frac{1}{r} \int_{\partial B_{r}} \operatorname{div}\left(u^{2} A x\right)= \\
& =-\frac{1}{r} \int_{\partial B_{r}} \mu u^{2}+\frac{1}{r} \int_{\partial B_{r}}(\operatorname{tr}(A)) u^{2}+\frac{1}{r} \int_{\partial B_{r}} u^{2} \partial_{x_{i}} a_{i j} x_{j}+\frac{2}{r} \int_{\partial B_{r}} u u_{x_{i}} a_{i j} x_{j},
\end{aligned}
$$

and, recalling that $A$ is symmetric, (2.21) follows.

Let us set

$$
I(r)=\int_{\partial B_{r}} u A \nabla u \cdot \nu=\int_{B_{r}} A \nabla u \cdot \nabla u \geq 0
$$

Lemma 2.6 There exists a positive constant $C$, only depending on $K$ and $L$, such that, for almost every $r \in(0, R)$,

$$
\begin{gathered}
\left|H^{\prime}(r)-\frac{n-1}{r} H(r)-2 I(r)\right| \leq \frac{C}{\rho_{0}} H(r), \\
I^{\prime}(r) \geq 2 \int_{\partial B_{r}} \frac{1}{\mu}(A \nabla u \cdot \nu)^{2}+\frac{n-2}{r} I(r)-\frac{C}{\rho_{0}} I(r),
\end{gathered}
$$

Proof For almost every $r \in(0, R)$, we can compute

$$
\begin{gathered}
H^{\prime}(r)-\frac{n-1}{r} H(r)-2 I(r)= \\
=\frac{1}{r} \int_{\partial B_{r}}\left(-A \nu \cdot \nu+\operatorname{tr}(A)+r \partial_{x_{i}} a_{i j} \nu_{j}\right) u^{2}-\frac{n-1}{r} \int_{\partial B_{r}} \mu u^{2}= \\
=\frac{1}{r} \int_{\partial B_{r}}(-A(0) \nu \cdot \nu+\operatorname{tr}(A(0))) u^{2}-\frac{n-1}{r} \int_{\partial B_{r}} \mu u^{2}+\frac{1}{r} \int_{\partial B_{r}} r \partial_{x_{i}} a_{i j} \nu_{j} u^{2}+ \\
+\frac{1}{r} \int_{\partial B_{r}}[-(A-A(0)) \nu \cdot \nu+(\operatorname{tr}(A)-\operatorname{tr}(A(0)))] u^{2}= \\
=\frac{n-1}{r} \int_{\partial B_{r}}(1-\mu) u^{2}+\frac{1}{r} \int_{\partial B_{r}} r \partial_{x_{i}} a_{i j} \nu_{j} u^{2}+ \\
\quad+\frac{1}{r} \int_{\partial B_{r}}[-(A-A(0)) \nu \cdot \nu+(\operatorname{tr}(A)-\operatorname{tr}(A(0)))] u^{2} .
\end{gathered}
$$

By (1.20) and (2.4), we have that

$$
\begin{gathered}
|(A(x)-A(0))| \leq \frac{L}{\rho_{0}}|x|, \\
|1-\mu(x)|=|(A(0)-A(x)) \nu \cdot \nu| \leq \frac{L}{\rho_{0}}|x|,
\end{gathered}
$$


and that the absolute values of the three terms in the right-hand side of (2.27) are bounded by $C H(r)$, obtaining (2.25).

Let us notice that

$$
I(r)=\int_{0}^{r} d s \int_{\partial B_{s}} A \nabla u \cdot \nabla u
$$

is continuously differentiable and that

$$
I^{\prime}(r)=\int_{\partial B_{r}} A \nabla u \cdot \nabla u
$$

Let us recall the following generalization of the Rellich identity [90], due to Payne and Weinberger [84]

$$
\begin{aligned}
& \int_{\partial B_{r}} a_{i j} \partial_{x_{i}} u \partial_{x_{j}} u f_{k} \nu_{k}= \\
&=2 \int_{\partial B_{r}} f_{i} a_{k j} \partial_{x_{i}} u \partial_{x_{j}} u \nu_{k}-2 \int_{B_{r}} f_{k} \partial_{x_{k}} u \partial_{x_{i}}\left(a_{i j} \partial_{x_{j}} u\right)+ \\
& \quad+\int_{B_{r}}\left(\partial_{x_{k}} f_{k} a_{i j}-2 \partial_{x_{k}} f_{i} a_{k j}+f_{k} \partial_{x_{k}} a_{i j}\right) \partial_{x_{i}} u \partial_{x_{j}} u
\end{aligned}
$$

which holds for every $f \in C^{0,1}\left(B_{r} ; \mathbb{R}^{n}\right)$ and every $u \in H^{2}\left(B_{r}\right)$, where $a_{i j}$ are as above. Choosing

$$
f(x)=\frac{A(x) x}{r \mu(x)}
$$

from (2.28) and (2.29), it follows that

$$
\left|\partial_{x_{i}} f_{j}-\frac{\delta_{i j}}{r}\right| \leq \frac{C}{\rho_{0}}
$$

where $C$ is a constant only depending on $L$. Inserting (2.33) in (2.32) and recalling (2.31) and (2.34), (2.26) easily follows.

The proof of Theorem 2.3 shall be based on a differential inequality for the so-called frequency function, a notion first introduced by Almgren [13], see also [32].

$$
N(r)=\frac{r I(r)}{H(r)}
$$

which is well-defined provided $H(r)>0$. Observe that the thesis of Theorem 2.3 is trivial if $u$ is identically constant in $B_{R}$, or, as is the same, if $I(R)=0$. Thus we may assume, with no loss of generality, that $I(R)>0$. Let us denote

$$
r^{\prime}=\inf \{r \in(0, R) \mid I(r)>0\},
$$

and observe that, being $I(r)$ increasing, we also have

$$
I(r)=0 \text { for every } r \leq r^{\prime},
$$




$$
\begin{gathered}
I(r)>0 \text { for every } r, r^{\prime}<r \leq R, \\
u(x) \equiv M, \text { for every } x \text { s.t. }|x| \leq r^{\prime},
\end{gathered}
$$

for some $M \in \mathbb{R}$.

Note that, by the maximum principle, if $H(\rho)=0$ for some $\rho \in(0, R)$, then $u \equiv 0$ in $B_{\rho}$ and $H(r)=0$ for every $r<\rho$. Therefore $H(r)>0$ and $N(r)$ is well-defined for every $r \in\left(r^{\prime}, R\right]$.

Lemma 2.7 There exists a positive constant $C$, only depending on $K$ and $L$, such that $e^{C \frac{r}{\rho_{0}}} N(r)$ is an increasing function on $\left(r^{\prime}, R\right)$.

Proof Let us recall that $I(r)$ is continuously differentiable in $(0, R)$ and that $H(r)$ is absolutely continuous in $(0, R)$, hence $N$ is absolutely continuous in $\left(r^{\prime}, R\right)$. If $r \in\left(r^{\prime}, R\right)$, recalling Lemma 2.6 and using Schwarz inequality, we compute

$$
\begin{aligned}
\frac{N^{\prime}(r)}{N(r)}=\frac{1}{r}+\frac{I^{\prime}(r)}{I(r)}-\frac{H^{\prime}(r)}{H(r)} & \geq \\
& \geq 2 \frac{\int_{\partial B_{r}} \frac{1}{\mu}(A \nabla u \cdot \nu)^{2}}{\int_{\partial B_{r}} u(A \nabla u \cdot \nu)}-2 \frac{\int_{\partial B_{r}} u(A \nabla u \cdot \nu)}{\int_{\partial B_{r}} \mu u^{2}}-\frac{C}{\rho_{0}} \geq-\frac{C}{\rho_{0}},
\end{aligned}
$$

where $C>0$ only depends on $K$ and $L$. Therefore

$$
N^{\prime}(r)+\frac{C}{\rho_{0}} N(r) \geq 0, \quad \text { for every } r \in\left(r^{\prime}, R\right),
$$

or, equivalently,

$$
\frac{d}{d r}\left(e^{C \frac{r}{\rho_{0}}} N(r)\right) \geq 0, \quad \text { for every } r \in\left(r^{\prime}, R\right),
$$

which proves the lemma.

Proof of Theorem 2.3 Let $r_{1}, r_{2}, r_{3}$ be such that $r^{\prime}<r_{1}<r_{2}<r_{3} \leq R$. By inequality (2.25) and by Lemma 2.7, we have

$$
\begin{aligned}
\log \frac{H\left(r_{2}\right)}{H\left(r_{1}\right)}=\int_{r_{1}}^{r_{2}} \frac{H^{\prime}(r)}{H(r)} d r & \leq(n-1) \log \frac{r_{2}}{r_{1}}+2 \int_{r_{1}}^{r_{2}} \frac{N(r)}{r} d r+\frac{C}{\rho_{0}}\left(r_{2}-r_{1}\right) \leq \\
& \leq(n-1) \log \frac{r_{2}}{r_{1}}+e^{\frac{C}{\rho_{0}}\left(r_{2}-r_{1}\right)} N\left(r_{2}\right) \log \frac{r_{2}}{r_{1}}+\frac{C}{\rho_{0}}\left(r_{2}-r_{1}\right), \\
\log \frac{H\left(r_{3}\right)}{H\left(r_{2}\right)}=\int_{r_{2}}^{r_{3}} \frac{H^{\prime}(r)}{H(r)} d r & \geq(n-1) \log \frac{r_{3}}{r_{2}}+2 \int_{r_{2}}^{r_{3}} \frac{N(r)}{r} d r-\frac{C}{\rho_{0}}\left(r_{3}-r_{2}\right) \geq \\
& \geq(n-1) \log \frac{r_{3}}{r_{2}}+e^{\frac{C}{\rho_{0}}\left(r_{2}-r_{3}\right)} N\left(r_{2}\right) \log \frac{r_{3}}{r_{2}}-\frac{C}{\rho_{0}}\left(r_{3}-r_{2}\right),
\end{aligned}
$$


where $C>0$ denotes a constant, only depending on $K$ and $L$. From (2.43) and (2.44), we have

$$
\begin{aligned}
& \log \frac{H\left(r_{2}\right)}{H\left(r_{1}\right)} \leq(n-1) \log \frac{r_{2}}{r_{1}}+ \\
& +e^{\frac{C}{\rho_{0}}\left(r_{3}-r_{1}\right)}\left(\frac{\log \frac{H\left(r_{3}\right)}{H\left(r_{2}\right)}}{\log \frac{r_{3}}{r_{2}}}-(n-1)+\frac{C\left(r_{3}-r_{2}\right)}{\rho_{0} \log \frac{r_{3}}{r_{2}}}\right) \log \frac{r_{2}}{r_{1}}+\frac{C}{\rho_{0}}\left(r_{2}-r_{1}\right) \leq \\
& \leq e^{\frac{C}{\rho_{0}}\left(r_{3}-r_{1}\right)} \frac{\log \frac{r_{2}}{r_{1}}}{\log \frac{r_{3}}{r_{2}}} \log \frac{H\left(r_{3}\right)}{H\left(r_{2}\right)}+\frac{C}{\rho_{0}}\left(r_{3}-r_{2}\right) e^{C\left(r_{3}-r_{1}\right)} \frac{\log \frac{r_{2}}{r_{1}}}{\log \frac{r_{3}}{r_{2}}}+\frac{C}{\rho_{0}}\left(r_{2}-r_{1}\right) \leq \\
& \leq p \log \frac{H\left(r_{3}\right)}{H\left(r_{2}\right)}+B
\end{aligned}
$$

where

$$
p=e^{C \max \left\{\frac{R}{\rho_{0}}, 1\right\}} \frac{\log \frac{r_{2}}{r_{1}}}{\log \frac{r_{3}}{r_{2}}},
$$

$$
B=C \max \left\{\frac{R}{\rho_{0}}, 1\right\}(1+p)
$$

and $C>0$ only depends on $K$ and $L$. We have

$$
\begin{gathered}
\frac{H\left(r_{2}\right)}{H\left(r_{1}\right)} \leq\left(\frac{H\left(r_{3}\right)}{H\left(r_{2}\right)}\right)^{p} e^{B}, \\
\left(H\left(r_{2}\right)\right)^{1+p} \leq e^{B} H\left(r_{1}\right)\left(H\left(r_{3}\right)\right)^{p}, \\
H\left(r_{2}\right) \leq e^{C \max \left\{\frac{R}{\rho_{0}}, 1\right\}}\left(H\left(r_{1}\right)\right)^{\frac{1}{1+p}}\left(H\left(r_{3}\right)\right)^{\frac{p}{1+p}} .
\end{gathered}
$$

Therefore, assuming the additional condition $r_{1}>r^{\prime}$, inequality (2.7) follows with $Q=$ $\exp \left(C \max \left\{\frac{R}{\rho_{0}}, 1\right\}\right)$ and $\alpha=\frac{1}{1+p}$.

It only remains to prove that $r^{\prime}=0$. Let us assume by contradiction that $r^{\prime}>0$. If we had $M=0$ in (2.39), then $H\left(r^{\prime}\right)=0$ and, passing to the limit in (2.7) as $r_{1} \rightarrow r^{\prime}$, it would follow $H(r)=0$ for every $r \in\left(r^{\prime}, R\right)$ and $u \equiv 0$ in $B_{R}$, leading to a contradiction with the definition (2.36) of $r^{\prime}$. Therefore in this case $r^{\prime}=0$. If $M \neq 0$ in (2.39), let us consider the function $v=u-M$, which satisfies $\nabla v \equiv \nabla u$ in $B_{R}$ and $v \equiv 0$ in $\overline{B_{r^{\prime}}}$. By applying the above arguments to $v$, we have again that $r^{\prime}=0$ and the proof is complete.

Remark 2.8 It is worth emphasizing once more the strength of the frequency function method just employed. In fact, besides the three-spheres inequality, it enables also to obtain a doubling inequality, and this was in fact the original purpose when the method was devised by Garofalo and Lin [32]. Let us outline here how the proof goes. For the sake of simplicity we stick to the normalized setting of Theorem 2.3. From the proof of Theorem 2.3 we have 
obtained that $r^{\prime}=0$ and, consequently, Lemma 2.7 applies for all $r \in(0, R)$. Hence, by (2.25) and by Lemma 2.7

$$
\begin{aligned}
\log \frac{H(2 r)}{H(r)}= & \int_{r}^{2 r} \frac{H^{\prime}(r)}{H(r)} d r \leq(n-1) \log 2+2 \int_{r}^{2 r} \frac{N(s)}{s} d s+\frac{C r}{\rho_{0}} \leq \\
& \leq(n-1) \log 2+2 \int_{r}^{2 r} e^{\frac{C}{\rho_{0}}(R-s)} \frac{N(R)}{s} d s+\frac{C r}{\rho_{0}} \leq \\
& \leq(n-1) \log 2+2 C N(R) \log 2+\frac{C R}{\rho_{0}}
\end{aligned}
$$

That is

$$
H(2 r) \leq Q H(r) \text {, for every } 0<r \leq \frac{R}{2}
$$

where $Q \geq 1$ only depends on $K, L, \frac{R}{\rho_{0}}$ and on $N(R)$. Consequently, by an integration and by the ellipticity bounds (1.19),

$$
\int_{B_{2 r}} u^{2} \leq Q \int_{B_{r}} u^{2}, \text { for every } 0<r \leq \frac{R}{2}
$$

with a possibly different $Q$, but still depending on the same quantities. As is well-known, (2.53) in turn implies the strong unique continuation property, because, by iteration on the radii $r_{n}=R 2^{-n}, n=1,2, \ldots$, we readily arrive at

$$
\int_{B_{r}} u^{2} \geq \frac{1}{Q}\left(\frac{r}{R}\right)^{\frac{\log Q}{\log 2}} \int_{B_{R}} u^{2}, \text { for every } 0<r \leq \frac{R}{2} .
$$

\section{The two-dimensional case}

In this section we shall deal with the two-dimensional case. We shall prove, by complexanalytic techniques, a three-spheres inequality for an elliptic equation in divergence form in pure principal part, see Theorem 3.11, The main difference with respect to higher dimensions is that in two dimensions we allow the coefficient matrix $A$ to be discontinuous. Actually here we allow $A$ to be also non-symmetric, however we stress that this hypothesis will be used in this section only.

Throughout this section we shall identify $z=x+\mathrm{i} y \in \mathbb{C}$ with points $(x, y) \in \mathbb{R}^{2}$ and we shall fix $A=A(z), z \in \mathbb{R}^{2}$, a real-valued $2 \times 2$ matrix such that its entries are measurable and it satisfies, for a given constant $K \geq 1$, the ellipticity condition

$$
\begin{aligned}
& A(z) \xi \cdot \xi \geq K^{-1}|\xi|^{2}, \quad \text { for almost every } z \in \mathbb{R}^{2}, \text { for every } \xi \in \mathbb{R}^{2} . \\
& A^{-1}(z) \xi \cdot \xi \geq K^{-1}|\xi|^{2},
\end{aligned}
$$

The constant $K$ will be referred to as the ellipticity constant of $A$. When $A$ is symmetric this condition coincides with the one given in (1.19). We also observe that (3.1) implies that

$$
\left|a_{i j}(z)\right| \leq \Lambda, \quad \text { for every } i, j=1,2 \text { and for almost every } z \in \mathbb{R}^{2} \text {, }
$$

$\Lambda$ depending on $K$ only. 
In this section, $\Omega$ will as usual denote a bounded connected open set contained in $\mathbb{R}^{2}$. Letting $f: \Omega \rightarrow \mathbb{C}$ be a complex-valued function, we shall make repeated use of the following notation for complex derivatives

$$
f_{\bar{z}}=\frac{1}{2}\left(f_{x}+\mathrm{i} f_{y}\right), \quad f_{z}=\frac{1}{2}\left(f_{x}-\mathrm{i} f_{y}\right) .
$$

We denote by $J=\left[\begin{array}{cc}0 & -1 \\ 1 & 0\end{array}\right]$ the counterclockwise rotation of $\frac{\pi}{2}$.

Proposition 3.1 Under the above stated hypotheses, let $u \in H_{\text {loc }}^{1}(\Omega)$ be a weak solution to the equation

$$
\operatorname{div}(A \nabla u)=0, \quad \text { in } \Omega,
$$

where $\Omega$ is a bounded simply connected open set in $\mathbb{R}^{2}$.

Then there exists a function $v \in H_{l o c}^{1}(\Omega)$ that satisfies

$$
\nabla v=J A \nabla u, \quad \text { almost everywhere in } \Omega .
$$

Moreover, letting $f=u+\mathrm{i} v$, we have

$$
f_{\bar{z}}=\mu f_{z}+\nu \overline{f_{z}}, \quad \text { almost everywhere in } \Omega \text {, }
$$

where $\mu$ and $\nu$ are bounded, measurable, complex-valued coefficients satisfying

$$
|\mu|+|\nu| \leq k<1, \quad \text { almost everywhere in } \Omega,
$$

$k$ being a constant depending on $K$ only.

On the other hand, if $f=u+\mathrm{i} v \in H_{\text {loc }}^{1}(\Omega, \mathbb{C})$ solves (3.4)-(3.5), then there exists a real-valued $2 \times 2$ matrix $A \in L^{\infty}(\Omega)$, satisfying (3.1) with a constant $K$ depending upon $k$ only, such that $u$ is a weak solution to (3.2).

Proof By (3.3) the vector $J A \nabla u$ is curl-free in the weak sense, thus $v$ is well-defined as long as $\Omega$ is simply connected, see [8, Theorem 2.1] for details. Then (3.4) follows from (3.3) with $\mu, \nu$ given by

$$
\begin{aligned}
& \mu=\frac{a_{22}-a_{11}-\mathrm{i}\left(a_{12}+a_{21}\right)}{a_{11} a_{22}-a_{12} a_{21}+a_{11}+a_{22}+1}, \\
& \nu=\frac{a_{12} a_{21}-a_{11} a_{22}+1+\mathrm{i}\left(a_{12}-a_{21}\right)}{a_{11} a_{22}-a_{12} a_{21}+a_{11}+a_{22}+1} .
\end{aligned}
$$

From these expressions and (3.1), one obtains, through elementary although lengthy computations, (3.5). In particular, $k$ is related to $K$ by the following formula

$$
k \leq \frac{K+\sqrt{K^{2}-1}-1}{K+\sqrt{K^{2}-1}+1} .
$$

This estimate is sharp and it is proved in [9]. 
Conversely, given the coefficients $\mu, \nu$ in (3.4) satisfying (3.5) one obtains (3.2) and (3.3) with $A$ given by

$$
A=\left[\begin{array}{cc}
\frac{|1-\mu|^{2}-|\nu|^{2}}{|1+\nu|^{2}-|\mu|^{2}} & \frac{2 \Im(\nu-\mu)}{|1+\nu|^{2}-|\mu|^{2}} \\
\frac{-2 \Im(\mu+\nu)}{|1+\nu|^{2}-|\mu|^{2}} & \frac{|1+\mu|^{2}-|\nu|^{2}}{|1+\nu|^{2}-|\mu|^{2}}
\end{array}\right]
$$

and the conclusion follows. It may be shown that the ellipticity constant of $A$ is related to $k$ by the following formula

$$
K \leq \frac{1+k}{1-k}
$$

Also this estimate is sharp, see again [9].

The function $v$ appearing above is usually called the stream function associated to $u$. Notice that $v$ is uniquely determined up to an additive constant and also that $v$ is a weak solution to

$$
\operatorname{div}(B \nabla v)=0, \quad \text { in } \Omega,
$$

where $B=(\operatorname{det} A)^{-1} A^{T}$, and where $\operatorname{det}(\cdot)$ denotes the determinant and $(\cdot)^{T}$ denotes the transpose. We notice that $B$ still satisfies the ellipticity condition (3.1), with the same ellipticity constant.

For any $k, 0 \leq k<1$, we say that a function $f: \Omega \rightarrow \mathbb{C}$ is a $k$-quasiconformal function in $\Omega$ if it is an $H_{l o c}^{1}(\Omega, \mathbb{C})$-solution to the Beltrami-type equation (3.4)-(3.5). A univalent solution to (3.4)-(3.5) is said a k-quasiconformal mapping. A function $f$ is a quasiconformal function, respectively mapping, if it is a k-quasiconformal function, respectively mapping, for some $k, 0 \leq k<1$. Concerning quasiconformal functions, their properties and characterizations we refer to [66].

Finally we observe that when $A$ is the identity matrix, then $u$ is harmonic and $v$ is simply its harmonic conjugate, thus $f=u+\mathrm{i} v$ is a holomorphic, or conformal, function, corresponding to $k=0$. Such a special case deserves our attention, especially when $\Omega=B_{1}$. We have the following result which links properties of a harmonic function $u$ to properties of its harmonic conjugate.

Proposition 3.2 Let $u$ be a harmonic function in $B_{1}$, and let $v$ be its harmonic conjugate normalized in such a way that $v(0)=0$.

Let us assume that $|u(z)|<E$ for every $|z|<1$. Then we have

$$
|v(z)| \leq \frac{2 E}{\pi} \log \left(\frac{1+|z|}{1-|z|}\right)
$$

If $u$ is Hölder continuous on $\overline{B_{1}}$, with Hölder exponent $\beta, 0<\beta<1$, then $v$ is also Hölder continuous on $\overline{B_{1}}$, with Hölder exponent $\beta$ and

$$
\|v\|_{C^{\beta}\left(\overline{B_{1}}\right)} \leq C_{0}\|u\|_{C^{\beta}\left(\overline{B_{1}}\right)}
$$

$C_{0}$ depending on $\beta$ only. 
Proof If $u$ is continuous up to the boundary of $B_{1}$, then

$$
f(z)=u(z)+\mathrm{i} v(z)=\frac{1}{2 \pi \mathrm{i}} \int_{\partial B_{1}} \frac{\xi+z}{\xi-z} u(\xi) \frac{d \xi}{\xi}, \quad \text { for every }|z|<1 .
$$

Such a representation is often referred to as Schwarz's formula or as Poisson representation. The inequality (3.9) follows immediately and it is sharp, take for instance $u=$ $\Im\left(\frac{2}{\pi} \log \left(\frac{1+z}{1-z}\right)\right)$ on $B_{1}$. The second result is due to Priwaloff, see [87].

Properties of harmonic functions and their conjugates, or of holomorphic functions, may be transferred to solutions to elliptic equations in divergence form and their stream functions, or to quasiconformal functions, through the following important representation theorem, a proof of which is due to Bers and Nirenberg [18], see also [17].

Theorem 3.3 Let $\Omega$ be a connected open set contained in $B_{1}$ and let $f \in H_{\text {loc }}^{1}(\Omega, \mathbb{C})$ solve (3.4) where $\mu, \nu$ satisfy (3.5).

Then there exist a $k$-quasiconformal mapping $\chi$ from $B_{1}$ onto itself and a holomorphic function $F$ on $\chi(\Omega)$ such that

$$
f=F \circ \chi
$$

We may choose $\chi$ such that $\chi(0)=0$. Moreover we have that the function $\chi$ and its inverse $\chi^{-1}$ satisfy the following Hölder continuity properties

$$
|\chi(x)-\chi(y)| \leq C_{1}|x-y|^{\beta}, \quad \text { for any } x, y \in B_{1}
$$

and

$$
\left|\chi^{-1}(x)-\chi^{-1}(y)\right| \leq C_{1}|x-y|^{\beta}, \quad \text { for any } x, y \in B_{1},
$$

where $C_{1}$ and $\beta, 0<\beta<1$, depend upon $k$ only.

Proof See [18, page 116] and [17].

Through the use of quasiconformal functions it is also possible to extend the classical method of harmonic measure in order to obtain a Hölder stability estimate in the interior for Cauchy problems for solutions to Beltrami-type equations (3.4)-(3.5). In turn, this provides a Hölder stability estimate in the interior for Cauchy problems for solutions to (3.2).

We denote by $\mathcal{L}_{A}$ the differential operator

$$
\mathcal{L}_{A} u=\operatorname{div}(A \nabla u) .
$$

We now introduce the notion of $\mathcal{L}_{A}$-harmonic measure $\omega$ of a subset $\Sigma$ of $\partial \Omega$. Roughly speaking $\omega$ is the solution to the Dirichlet problem

$$
\begin{cases}\operatorname{div}(A \nabla \omega)=0, & \text { in } \Omega, \\ \omega=1, & \text { on } \Sigma, \\ \omega=0, & \text { on } \partial \Omega \backslash \Sigma .\end{cases}
$$

However, being the set $\Sigma$ arbitrary, and since the Dirichlet data in (3.14) may be discontinuous, a more careful definition is needed. Let us recall some notions from potential theory, see for instance [41]. 
Definition 3.4 A function $u: \Omega \mapsto \mathbb{R} \cup\{+\infty\}$ is called $\mathcal{L}_{A^{-}}$superharmonic in $\Omega$ if

(i) $u$ is lower semicontinuous;

(ii) $u \not \equiv+\infty$ in any connected component of $\Omega$;

(iii) for any open set $\Omega_{1} \subset \subset \Omega$ and any $h \in C\left(\overline{\Omega_{1}}\right)$, such that $\mathcal{L}_{A} h=0$ in the weak sense in $\Omega_{1}$, if $u \geq h$ on $\partial \Omega_{1}$ then $u \geq h$ in $\Omega_{1}$.

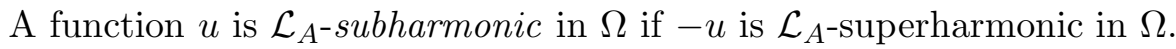

Definition 3.5 Let $\Sigma$ be a subset of $\partial \Omega$ and let $\chi_{\Sigma}$ be its characteristic function. We define $\mathcal{U}_{\Sigma}$ as the class of the $\mathcal{L}_{A^{-}}$-superharmonic functions $u$ in $\Omega$ such that $u \geq 0$ and $\liminf _{x \rightarrow y} u(x) \geq \chi_{\Sigma}(y)$ for any $y \in \partial \Omega$.

We define the $\mathcal{L}_{A}$-harmonic measure of $\Sigma$ with respect to $\Omega$ as the upper Perron solution with respect to $\chi_{\Sigma}$, that is

$$
\omega(z)=\omega\left(\Sigma, \Omega, \mathcal{L}_{A} ; z\right)=\inf \left\{u(z) \mid u \in \mathcal{U}_{\Sigma}\right\}, \quad \text { for any } z \in \Omega .
$$

We observe that we have $0 \leq \omega \leq 1$ everywhere. In order to have that $0<\omega<1$ in $\Omega$, we need to guarantee that $\omega$ is not identically equal to 0 or to 1 . A simple sufficient condition is the following, [41, Theorem 11.6]. We recall that a continuum is a closed connected set with at least two points. There exist two continua, $\gamma_{1}$ and $\gamma_{2}$, such that $\gamma_{1}$ is contained in $\Sigma$ and has positive distance from $\partial \Omega \backslash \Sigma$ and $\gamma_{2}$ is contained in $\partial \Omega \backslash \Sigma$ and has positive distance from $\Sigma$. For a more thorough analysis of this question we refer to [41].

Lemma 3.6 Let $f \in H_{l o c}^{1}(\Omega, \mathbb{C})$ be a non-identically zero solution to (3.4)-(3.5). Then there exists a real-valued $2 \times 2$ matrix $A_{1} \in L^{\infty}(\Omega)$ such that $A_{1}$ satisfies (3.1) with a constant $K$ depending on $k$ only, and $\varphi=\log |f|$ is $\mathcal{L}_{A_{1}}$-subharmonic.

Proof We denote

$$
\mu_{1}= \begin{cases}\mu, & \text { where } f_{z}=0 \\ \mu+\nu \overline{f_{z}} / f_{z}, & \text { where } f_{z} \neq 0 .\end{cases}
$$

Then we have that $\left|\mu_{1}\right| \leq k$ almost everywhere in $\Omega$ and $f$ satisfies

$$
f_{\bar{z}}=\mu_{1} f_{z}, \quad \text { almost everywhere in } \Omega .
$$

Let $z$ be a point in $\Omega$ such that $f(z) \neq 0$. Locally, on a neighbourhood of $z$, we can define the function $\Phi=\log f$ where $\log$ is any possible determination of the logarithm in the complex plane. In this neighbourhood $\Phi$ also satisfies

$$
\Phi_{\bar{z}}=\mu_{1} \Phi_{z}
$$

Then by Proposition 3.1 the function $\varphi=\log |f|=\Re \log f$ locally satisfies

$$
\operatorname{div}\left(A_{1} \nabla \varphi\right)=0
$$

where the matrix $A_{1}$ is given by (3.7) with $\mu$ and $\nu$ replaced by $\mu_{1}$ and 0 , respectively. Note incidentally that $A_{1}$ is symmetric, that is $A_{1}=A_{1}^{T}$, $\operatorname{det} A_{1}=1$, and hence $B_{1}=$ $\left(\operatorname{det} A_{1}\right)^{-1} A_{1}^{T}=A_{1}$. 
We remark that we can define $\varphi=\log |f|$ globally as an $H_{l o c}^{1}\left(\Omega_{1}\right)$ function, where $\Omega_{1}=\{z \in \Omega \mid f(z) \neq 0\}$, hence using a partition of unity it is easy to show that (3.16) holds weakly in $\Omega_{1}$.

Clearly, by Theorem 3.3, the set $\{z \in \Omega \mid f(z)=0\}$ consists of isolated points and $\varphi$ goes uniformly to $-\infty$ as $z$ converges to an element of such a set.

Using this remark and the maximum principle we can prove in an elementary way that $\varphi=\log |f|$ is $\mathcal{L}_{A_{1}}$-subharmonic.

We observe that if $k=0$, that is $f$ is holomorphic, then $A_{1}$ in the previous lemma is the identity matrix. By the use of the $\mathcal{L}_{A_{1}}$-harmonic measure we obtain a Hölder stability estimate in the interior for Cauchy problems for a Beltrami-type equation (3.4)-(3.5)).

Theorem 3.7 Let $\Sigma$ be a subset of $\partial \Omega$. Let $f \in H_{l o c}^{1}(\Omega, \mathbb{C})$ solve (3.4)-(3.5).

If $\|f\|_{L^{\infty}(\Omega)} \leq E$ and we have that, given $\eta>0$,

$$
\limsup _{x \rightarrow y}|f(x)| \leq \eta, \quad \text { for any } y \in \Sigma,
$$

then for any $z \in \Omega$ the following estimate holds

$$
|f(z)| \leq E^{1-\omega(z)} \eta^{\omega(z)},
$$

where $\omega=\omega\left(\Sigma, \Omega, \mathcal{L}_{A_{1}}\right)$ is the $\mathcal{L}_{A_{1}}$-harmonic measure of $\Sigma$ with respect to $\Omega$ and the matrix $A_{1}$ is defined as in Lemma 3.6.

Proof This result was obtained in [10, Theorem 4.5]. We sketch a proof, for the sake of completeness. It is evident that $0<\eta \leq E$. If $\eta=E$ the proof is trivial. We consider then the case $0<\eta<E$. By Lemma 3.6 the function $\varphi=\log |f|$ is $\mathcal{L}_{A_{1}}$-subharmonic. Let $\omega=\omega\left(\Sigma, \Omega, \mathcal{L}_{A_{1}}\right)$ be the $\mathcal{L}_{A_{1}}$-harmonic measure of $\Sigma$ with respect to $\Omega$.

Let us denote

$$
\widetilde{\varphi}=\frac{\log \left(\frac{|f|}{E}\right)}{\log \left(\frac{\eta}{E}\right)} .
$$

It is easy to see that $\widetilde{\varphi}$ belongs to the upper class $\mathcal{U}_{\Sigma}$. Hence for any $z \in \Omega$ we have $\omega(z) \leq \widetilde{\varphi}(z)$ and consequently

$$
\varphi(z) \leq \log (\eta) \omega(z)+\log (E)(1-\omega(z))
$$

Thus the conclusion follows.

We observe that, in view of Proposition 3.1, the above Theorem 3.7 could be restated in terms of a Cauchy problem for an elliptic equation like (3.2).

Theorem 3.8 Let $\Sigma$ be a subset of $\partial \Omega$. Let $u \in H^{1}(\Omega)$ be a solution to (3.2). Let us assume that there exists a single-valued stream function $v$ in $\Omega$ and let $f=u+\mathrm{i} v$. We a-priori assume that $\|f\|_{L^{\infty}(\Omega)} \leq E$.

We assume that $\Sigma \subset \partial \Omega$ is a connected open Lipschitz arc of $\partial \Omega$ and as usual we denote $g=\left.u\right|_{\Sigma}$ and $\psi=\left.A \nabla u \cdot \nu\right|_{\Sigma}$ the Cauchy data of $u$ on $\Sigma$. We suppose that $g \in C^{0}(\Sigma)$ and $\psi \in L^{1}(\Sigma)$. 
If, given $\eta>0$,

$$
\|g\|_{L^{\infty}(\Sigma)}+\|\psi\|_{L^{1}(\Sigma)} \leq \eta
$$

then for any $z \in \Omega$ the following estimate holds

$$
|u(z)| \leq E^{1-\omega(z)} \eta^{\omega(z)}
$$

where $\omega=\omega\left(\Sigma, \Omega, \mathcal{L}_{A_{1}}\right)$ is the $\mathcal{L}_{A_{1}}$-harmonic measure of $\Sigma$ with respect to $\Omega$ and the matrix $A_{1}$ is defined as in Lemma 3.6 with $\mu$ and $\nu$ given by (3.6).

Proof By our assumptions on $g$ and $\psi$, and classical regularity estimates, we have that $f$ is continuous at any point $y \in \Sigma$.

We normalize $v$ in such a way that $v=0$ on some point of $\Sigma$, and we infer that $\|f\|_{L^{\infty}(\Sigma)} \leq \eta$ and, in view of the regularity of $f$, we obtain that (3.17) holds. Therefore the conclusion is an immediate consequence of Theorem 3.7.

It is evident that in order to make such estimates practically useful, it is necessary to provide a positive lower bound on $\omega$ and this is usually obtained by using the Harnack inequality for positive solutions to elliptic equations in divergence form, [34, Theorem 8.20], see for details [10].

Our principal aim here is however to concentrate on three-spheres inequalities, first for holomorphic and quasiconformal functions, then for harmonic functions and solutions to elliptic equations in divergence form.

Theorem 3.9 Let $R>0$ and $0 \leq k<1$, and let $f$ be a $k$-quasiconformal function on $B_{R}$. Then for every $r_{1}, r_{2}, r_{3}$, with $0<r_{1}<r_{2}<r_{3} \leq R$, there exists a constant $\alpha, 0<\alpha<1$, depending on $k, \frac{r_{2}}{r_{1}}$ and $\frac{r_{3}}{r_{2}}$ only, such that

$$
\|f\|_{L^{\infty}\left(B_{r_{2}}\right)} \leq\|f\|_{L^{\infty}\left(B_{r_{1}}\right)}^{\alpha}\|f\|_{L^{\infty}\left(B_{r_{3}}\right)}^{1-\alpha} .
$$

Proof We may use the previous Theorem 3.7 , by setting $\Omega=B_{r_{3}} \backslash \overline{B_{r_{1}}}$ and $\Sigma=\partial B_{r_{1}} \subset \partial \Omega$. Then

$$
\alpha=\inf _{z \in B_{r_{2}} \backslash \overline{B_{r_{1}}}} \omega\left(\Sigma, \Omega, \mathcal{L}_{A_{1}}\right)(z) .
$$

For example, when $k=0$, by this technique we may recover the three-circles theorem for holomorphic functions by Hadamard. In fact, in this case $A_{1}$ is the identity matrix and by explicit computations in radial coordinates we obtain

$$
\alpha=\frac{\log \left(\frac{r_{3}}{r_{2}}\right)}{\log \left(\frac{r_{3}}{r_{1}}\right)}, \quad \text { when } k=0 .
$$

In order to obtain an essentially optimal value of $\alpha$ in the general case, that is when $0 \leq k<1$, we use the representation theorem, Theorem 3.3. A related argument was used already in [7, Proposition 1]. Up to a linear change of variables, we may temporarily assume that $r_{3}=1$. We apply Theorem 3.3, then $f=F \circ \chi$, where $F$ is conformal on $B_{1}$ and $\chi$ is a quasiconformal mapping between $B_{1}$ and itself. Let $C_{1}$ and $\beta, 0<\beta<1$, be the constants appearing in (3.11) and (3.12), which characterize the Hölder continuity of the quasiconformal mapping $\chi$ and of its inverse. Then there exist $0<\widetilde{r}_{1}<\widetilde{r}_{2}<1$ such that 
$B_{\widetilde{r}_{1}} \subset \chi\left(B_{r_{1}}\right)$ and $\chi\left(B_{r_{2}}\right) \subset B_{\widetilde{r}_{2}}$ or, as is the same, $\chi\left(B_{1} \backslash \overline{B r_{2}}\right) \supset B_{1} \backslash \overline{B_{\widetilde{r}_{2}}}$. We apply the three-circles theorem to $F$, and we obtain that (3.21) holds with

$$
\alpha=\frac{\log \left(\frac{1}{\widetilde{r}_{2}}\right)}{\log \left(\frac{1}{\widetilde{r}_{1}}\right)}, \quad \text { when } 0 \leq k<1,
$$

where we may take

$$
\widetilde{r}_{1}=\left(\frac{r_{1}}{C_{1} r_{3}}\right)^{1 / \beta} \text { and } \widetilde{r}_{2}=1-\left(\frac{1-\frac{r_{2}}{r_{3}}}{C_{1}}\right)^{1 / \beta}
$$

$C_{1}$ and $\beta, 0<\beta<1$, depending on $k$ only. The above choices of $\widetilde{r}_{1}$ and $\widetilde{r}_{2}$ serve our purposes for any $r_{3}>0$.

We now turn our attention to the second order elliptic equation. We shall prove a threespheres inequality first for $L^{\infty}$-norms and then for $L^{2}$-norms. Let $R>0$ and let $u \in H_{l o c}^{1}\left(B_{R}\right)$ solve

$$
\operatorname{div}(A \nabla u)=0, \quad \text { in } B_{R}
$$

Theorem 3.10 Under the above stated hypotheses, for every $r_{1}, r_{2}, r_{3}$, with $0<r_{1}<r_{2}<$ $r_{3} \leq R$, there exist constants $Q \geq 1$ and $\alpha, 0<\alpha<1$, depending on $K, \frac{r_{2}}{r_{1}}$ and $\frac{r_{3}}{r_{2}}$ only, such that

$$
\|u\|_{L^{\infty}\left(B_{r_{2}}\right)} \leq Q\|u\|_{L^{\infty}\left(B_{r_{1}}\right)}^{\alpha}\|u\|_{L^{\infty}\left(B_{r_{3}}\right)}^{1-\alpha} .
$$

Proof We temporarily assume that $r_{3}=1$ and also $A=I d$, that is $u$ harmonic in $B_{1}$. We let $v$ be its harmonic conjugate normalized in such a way that $v(0)=0$. By (3.9), we may bound the values of $v$ in terms of those on $u$. For example, we have

$$
\|v\|_{L^{\infty}\left(B_{\frac{r_{1}}{2}}\right)} \leq C_{2}\|u\|_{L^{\infty}\left(B_{r_{1}}\right)} \quad \text { and } \quad\|v\|_{L^{\infty}\left(B_{\frac{r_{2}+1}{2}}\right)} \leq C_{3}\|u\|_{L^{\infty}\left(B_{1}\right)}
$$

with

$$
C_{2}=\frac{2}{\pi} \log 3 \quad \text { and } \quad C_{3}=\frac{2}{\pi} \log \left(\frac{3+\frac{r_{2}}{r_{3}}}{1-\frac{r_{2}}{r_{3}}}\right) .
$$

Therefore, we may use the Hadamard three-circles theorem and conclude that for $u$ harmonic in $B_{R}$ we have

$$
\|u\|_{L^{\infty}\left(B_{r_{2}}\right)} \leq\left(\left(C_{3}+1\right)\|u\|_{L^{\infty}\left(B_{r_{3}}\right)}\right)^{1-\alpha}\left(\left(C_{2}+1\right)\|u\|_{L^{\infty}\left(B_{r_{1}}\right)}\right)^{\alpha}
$$

with $\alpha$ given by

$$
\alpha=\frac{\log \left(\frac{1}{2}+\frac{r_{3}}{2 r_{2}}\right)}{\log \left(\left(1+\frac{r_{2}}{r_{3}}\right) \frac{r_{3}}{r_{1}}\right)} .
$$

For the general case of solutions to elliptic equations in divergence form, we use the same argument as in Theorem 3.9. By Proposition 3.1 and Theorem 3.3, we have that $u=U \circ \chi$ 
where $U$ is a harmonic function in $B_{1}$ and $\chi$ is a quasiconformal mapping between $B_{1}$ and itself. Then we apply (3.27) to $U$ and we conclude that (3.25) is satisfied in the following form. It holds (3.27) under conditions (3.26), (3.28), with $\frac{r_{1}}{r_{3}}$ and $\frac{r_{2}}{r_{3}}$ replaced by $\widetilde{r}_{1}$ and $\widetilde{r}_{2}$, respectively, $\widetilde{r}_{1}$ and $\widetilde{r}_{2}$ given by (3.23).

We remark that for harmonic functions, instead of (3.27), we might have used a result by Korevaar and Meyers, [56]. They proved that for $u$ harmonic in $B_{R}$ a three-spheres inequality holds with $Q=1$, that is

$$
\|u\|_{L^{\infty}\left(B_{r_{2}}\right)} \leq\|u\|_{L^{\infty}\left(B_{r_{3}}\right)}^{1-\widetilde{\alpha}}\|u\|_{L^{\infty}\left(B_{r_{1}}\right)}^{\widetilde{\alpha}},
$$

where

$$
\widetilde{\alpha}=\widetilde{\alpha}\left(\frac{r_{2}}{r_{1}}, \frac{r_{3}}{r_{2}}\right)
$$

We observe that their result is actually valid in any dimension $n \geq 2$. Let us also recall an interesting related result by Petrosyan [85].

As a corollary to Theorem 3.11 we obtain the corresponding three-spheres inequality in $L^{2}$-norms as well.

Theorem 3.11 (Three-spheres inequality - two-dimensional case) Under the previously stated hypotheses, for every $r_{1}, r_{2}, r_{3}$, with $0<r_{1}<r_{2}<r_{3} \leq R$, there exist constants $Q \geq 1$ and $\alpha, 0<\alpha<1$, depending on $K, \frac{r_{2}}{r_{1}}$ and $\frac{r_{3}}{r_{2}}$ only, such that

$$
\|u\|_{L^{2}\left(B_{r_{2}}\right)} \leq Q\|u\|_{L^{2}\left(B_{r_{1}}\right)}^{\alpha}\|u\|_{L^{2}\left(B_{r_{3}}\right)}^{1-\alpha} .
$$

Proof We use the local boundedness estimate for weak solutions to (3.24), see for instance [34, Theorem 8.17], telling that for a constant $\widetilde{C}$ depending on $K$ only, and for every $s, r$, $0<s<r \leq R$, we have

$$
\|u\|_{L^{\infty}\left(B_{s}\right)} \leq \frac{\widetilde{C} \rho_{0}}{r-s}\|u\|_{L^{2}\left(B_{r}\right)} .
$$

We apply this bound with the following choices of $s, r$

$$
s=\frac{r_{2}+r_{3}}{2}, \quad r=r_{3},
$$

and also

$$
s=\frac{r_{1}}{2}, \quad r=r_{1},
$$

in combination with Theorem 3.10 with radii $0<\frac{r_{1}}{2}<r_{2}<\frac{r_{2}+r_{3}}{2}$. Hence, by the trivial estimate

$$
\|u\|_{L^{2}\left(B_{r_{2}}\right)} \leq \frac{\sqrt{\pi} r_{2}}{\rho_{0}}\|u\|_{L^{\infty}\left(B_{r_{2}}\right)}
$$

we conclude that

$$
\|u\|_{L^{2}\left(B_{r_{2}}\right)} \leq \sqrt{\pi} Q \widetilde{C}\left(\frac{4 r_{2}}{r_{3}-r_{2}}\|u\|_{L^{2}\left(B_{r_{3}}\right)}\right)^{1-\alpha}\left(\frac{2 r_{2}}{r_{1}}\|u\|_{L^{2}\left(B_{r_{1}}\right)}\right)^{\alpha}
$$

where $Q$ and $\alpha$ are the constants appearing in (3.25) related to $K, \frac{2 r_{2}}{r_{1}}$ and $\frac{r_{2}+r_{3}}{2 r_{2}}$. 


\section{The three-spheres inequality for the complete equation}

Here and in the rest of the paper we consider the elliptic operator $\operatorname{div}(A \nabla \cdot)+c \cdot$ where $A$ and $c$ satisfy the conditions (1.19), (1.20), and (1.21), respectively, stated in the Introduction. We recall that the Lipschitz condition (1.20) is invoked only when $n \geq 3$.

We start by considering solutions $u$ to the homogeneous equation

$$
\operatorname{div}(A \nabla u)+c u=0, \quad \text { in } B_{R} .
$$

Theorem 4.1 (Three-spheres inequality - equation with zero order term) If the above stated hypotheses hold, there exists $C_{0}, 0<C_{0} \leq 1$, only depending on $K, L$ and $\kappa$, such that, setting

$$
R_{0}=\min \left\{R, C_{0} \rho_{0}\right\}
$$

for every $r_{1}, r_{2}, r_{3}$, with $0<r_{1}<r_{2}<\frac{r_{3}}{4 K} \leq r_{3} \leq R_{0}$,

$$
\|u\|_{L^{2}\left(B_{r_{2}}\right)} \leq Q\|u\|_{L^{2}\left(B_{r_{1}}\right)}^{\alpha}\|u\|_{L^{2}\left(B_{r_{3}}\right)}^{1-\alpha}
$$

where $Q \geq 1$ and $\alpha, 0<\alpha<1$ only depend on $K, L, \kappa, \max \left\{\frac{R}{\rho_{0}}, 1\right\}, \frac{r_{2}}{r_{1}}, \frac{r_{3}}{r_{2}}$.

The thesis is an immediate consequence of the following two lemmas.

Lemma 4.2 For every $\delta>0$ there exists $C_{0}, 0<C_{0} \leq 1$, only depending on $K, L, \kappa$ and $\delta$ such that, denoting $R_{0}=\min \left\{R, C_{0} \rho_{0}\right\}$, there exists a positive solution $w \in C^{1}\left(B_{R_{0}}\right)$ to

$$
\operatorname{div}(A \nabla w)+c w=0, \quad \text { in } B_{R_{0}}
$$

such that

$$
\frac{1}{1+\delta^{2}} \leq w \leq 1+\delta^{2}, \quad \text { in } B_{R_{0}}
$$

and when $n \geq 3$

$$
|\nabla w| \leq \frac{\delta}{\rho_{0}}, \quad \text { in } B_{R_{0}}
$$

Proof There exists $C_{1}>0$ only depending on $K, \kappa$, such that the operator $\operatorname{div}(A \nabla \cdot)+c \cdot$ is coercive on $H_{0}^{1}\left(B_{r}\right)$ for every $r \leq R_{1}$, with $R_{1}=\min \left\{R, C_{1} \rho_{0}\right\}$, see for instance [34, Lemma 8.4]. Let us pick the unique solution $w$ to

$$
\begin{cases}\operatorname{div}(A \nabla w)+c w=0, & \text { in } B_{r}, \\ w=1, & \text { on } \partial B_{r} .\end{cases}
$$

Denoting $z=w-1$, we have

$$
\begin{cases}\operatorname{div}(A \nabla z)=-c(1+z), & \text { in } B_{r}, \\ z=0, & \text { on } \partial B_{r} .\end{cases}
$$


By standard a-priori bounds in $L^{\infty}$, see [34, Theorem 8.16], we have

$$
\|z\|_{L^{\infty}\left(B_{r}\right)} \leq C \kappa \frac{r^{2}}{\rho_{0}^{2}}\left(1+\|z\|_{L^{\infty}\left(B_{r}\right)}\right)
$$

where $C$ only depends on $K$. Hence there exists $C_{2} \leq C_{1}$ only depending on $K, \kappa$, such that for every $r \leq R_{2}$, with $R_{2}=\min \left\{R, C_{2} \rho_{0}\right\}$ we have

$$
\|z\|_{L^{\infty}\left(B_{r}\right)} \leq C \kappa \frac{r^{2}}{\rho_{0}^{2}}
$$

Next, when $n \geq 3$, applying to (4.8) a global Schauder type estimate, see [34, Theorem 8.33], one obtains, for every $r \leq R_{2}$,

$$
\|\nabla z\|_{L^{\infty}\left(B_{r}\right)} \leq C \kappa \frac{r}{\rho_{0}^{2}}\left(1+\|z\|_{L^{\infty}\left(B_{r}\right)}\right),
$$

where $C$ only depends on $K, L$. Thus, we may find $C_{0} \leq C_{2}$ only depending on $K, \kappa, L$ and $\delta$, such that, denoting $R_{0}=\min \left\{R, C_{0} \rho_{0}\right\}$, we have

$$
\|z\|_{L^{\infty}\left(B_{R_{0}}\right)} \leq \frac{\delta^{2}}{1+\delta^{2}}
$$

and when $n \geq 3$

$$
\|\nabla z\|_{L^{\infty}\left(B_{R_{0}}\right)} \leq \frac{\delta}{\rho_{0}}
$$

Therefore (4.5) and (4.6) follow immediately.

Observe that, for the sake of simplicity and with no loss of generality, we can also assume $C_{0} \leq 1$.

Lemma 4.3 Let $R_{0}$ be the quantity introduced in Lemma 4.2. when $\delta$ is chosen to be $\delta=1$ when $n=2$ and $\delta=\min \left\{\frac{L}{K}, 1\right\}$ when $n \geq 3$. Then $u$ can be factored in $B_{R_{0}}$ as

$$
u=w v,
$$

where $w$ is the function constructed in Lemma 4.2 and $v$ solves

$$
\operatorname{div}(\widetilde{A} \nabla v)=0, \quad \text { in } B_{R_{0}},
$$

where the matrix $\widetilde{A}$ is given by

$$
\widetilde{A}=w^{2} A,
$$

and it satisfies

$$
\frac{1}{4 K}|\xi|^{2} \leq \widetilde{A}(x) \xi \cdot \xi \leq 4 K|\xi|^{2}, \quad \text { for almost every } x \in B_{R_{0}} \text {, for every } \xi \in \mathbb{R}^{n} .
$$

and when $n \geq 3$

$$
|\widetilde{A}(x)-\widetilde{A}(y)| \leq \frac{8 L}{\rho_{0}}|x-y|, \quad \text { for every } x, y \in B_{R_{0}}
$$


Proof The proof is straightforward.

Let us now consider the inhomogeneous elliptic equation

$$
\operatorname{div}(A \nabla u)+c u=f+\operatorname{div} F, \quad \text { in } B_{R},
$$

where $f \in L^{2}\left(\mathbb{R}^{n}\right)$ and $F \in L^{2}\left(\mathbb{R}^{n} ; \mathbb{R}^{n}\right)$ satisfy (1.29).

Theorem 4.4 (Three-spheres inequality - complete equation) If the the above stated hypotheses hold, for every $r_{1}, r_{2}, r_{3}$, with $0<r_{1}<r_{2}<\frac{r_{3}}{4 K} \leq r_{3} \leq R_{0}$,

$$
\|u\|_{L^{2}\left(B_{r_{2}}\right)} \leq Q\left(\|u\|_{L^{2}\left(B_{r_{1}}\right)}+\varepsilon\right)^{\alpha}\left(\|u\|_{L^{2}\left(B_{r_{3}}\right)}+\varepsilon\right)^{1-\alpha}
$$

where $Q \geq 1$ and $\alpha, 0<\alpha<1$, only depend on $K, L, \kappa, \max \left\{\frac{R}{\rho_{0}}, 1\right\}, \frac{r_{2}}{r_{1}}, \frac{r_{3}}{r_{2}}$, and $R_{0}$ is given by (4.2).

Proof Let us consider the unique solution $u_{0}$ to

$$
\begin{cases}\operatorname{div}\left(A \nabla u_{0}\right)+c u_{0}=f+\operatorname{div} F, & \text { in } B_{R_{0}}, \\ u_{0}=0, & \text { on } \partial B_{R_{0}} .\end{cases}
$$

We have that

$$
\left\|u_{0}\right\|_{L^{2}\left(B_{R_{0}}\right)} \leq C\left(R_{0}^{2}\|f\|_{L^{2}\left(\mathbb{R}^{n}\right)}+R_{0}\|F\|_{L^{2}\left(\mathbb{R}^{n} ; \mathbb{R}^{n}\right)}\right)
$$

with $C$ only depending on $K$. Noticing that $u-u_{0}$ satisfies the hypotheses of Theorem 4.1 , the thesis follows immediately.

\section{Propagation of smallness}

For every $G \subset \mathbb{R}^{n}$ and for every $h>0$ we shall denote

$$
\begin{aligned}
& G_{h}=\{x \in G \mid \operatorname{dist}(x, \partial G)>h\}, \\
& G^{h}=\left\{x \in \mathbb{R}^{n} \mid \operatorname{dist}(x, \bar{G})<h\right\} .
\end{aligned}
$$

Theorem 5.1 (Propagation of smallness in the interior) Let $\Omega$ be a bounded connected open set in $\mathbb{R}^{n}$, and let $B_{r_{0}}\left(x_{0}\right) \subset \Omega$ be a fixed ball. Let $C_{0}$ be as in the thesis of Theorem 4.1. Let $h, 0<h \leq \min \left\{2 C_{0} \rho_{0}, \frac{r_{0}}{2}\right\}$, be fixed and let $G \subset \Omega$ be a connected open set such that $\operatorname{dist}(G, \partial \Omega) \geq h$ and $B_{\frac{r_{0}}{2}}\left(x_{0}\right) \subset G$.

Let $u \in H_{\text {loc }}^{1}(\Omega)$ be a solution to the equation

$$
\operatorname{div}(A \nabla u)+c u=f+\operatorname{div} F, \quad \text { in } \Omega,
$$

where $f$ and $F$ satisfy (1.29). Let us assume that

$$
\|u\|_{L^{2}\left(B_{r_{0}}\left(x_{0}\right)\right)} \leq \eta,
$$




$$
\|u\|_{L^{2}(\Omega)} \leq E_{0}
$$

for given $\eta>0, E_{0}>0$. We have

$$
\|u\|_{L^{2}(G)} \leq C(\eta+\varepsilon)^{\delta}\left(E_{0}+\varepsilon\right)^{1-\delta},
$$

where

$$
C=C_{1}\left(\frac{|\Omega|}{h^{n}}\right)^{\frac{1}{2}}
$$

and

$$
\delta \geq \alpha^{\frac{C_{2}|\Omega|}{h^{n}}}
$$

with $C_{1}>0$ and $\alpha, 0<\alpha<1$, only depending on $K, L$ and $\kappa$ and $C_{2}$ only depending on $K$.

Proof We shall need uniform three-spheres inequalities in a domain slightly larger than $G$. For instance, for any $x \in G^{\frac{h}{2}}$, we have $B_{\frac{h}{2}}(x) \subset \Omega$. Therefore we can apply the three-spheres inequality (4.19) to spheres centered at $x$ choosing $R=h / 2$. Hence $R_{0}=\min \left\{\frac{h}{2}, C_{0} \rho_{0}\right\}$ and, by our choice in the assumptions, $R_{0}=\frac{h}{2}$. Moreover, recalling that $C_{0} \leq 1$, we also have $\max \left\{\frac{R_{0}}{\rho_{0}}, 1\right\}=1$.

Next we can fix radii $r_{1}, r_{2}, r_{3}$ as follows

$$
r_{3}=\frac{h}{2}, \quad r_{2}=\frac{r_{3}}{5 K}=\frac{h}{10 K}, \quad r_{1}=\frac{1}{3} r_{2}=\frac{h}{30 K} .
$$

With such a choice the inequality (4.19) applies with $Q \geq 1$ and $\alpha, 0<\alpha<1$, only depending on $K, L$ and $\kappa$.

Let us consider the set $G^{r_{1}}$ as defined in (5.2). We have that $G^{r_{1}}$ is a connected open set containing $G$ such that $\operatorname{dist}\left(G^{r_{1}}, \partial \Omega\right) \geq h-r_{1}>r_{3}=\frac{h}{2}$. For every $y \in G^{r_{1}}$, there exists a continuous path $\gamma:[0.1] \rightarrow G^{r_{1}}$ such that $\gamma(0)=x_{0}, \gamma(1)=y$. Let us define $0=t_{0}<$ $t_{1}<\ldots<t_{N}=1$, according to the following rule. We set $t_{k+1}=\max \left\{t|| \gamma(t)-x_{k} \mid=2 r_{1}\right\}$ if $\left|x_{k}-y\right|>2 r_{1}$, otherwise we stop the process and set $N=k+1, t_{N}=1$. Let $x_{k}=\gamma\left(t_{k}\right)$. The balls $B_{r_{1}}\left(x_{k}\right)$ are pairwise disjoint for $k=0, \ldots, N-1$ and $\left|x_{k+1}-x_{k}\right|=2 r_{1}$. Since $r_{2}=3 r_{1}$ we have that $B_{r_{1}}\left(x_{k+1}\right) \subset B_{r_{2}}\left(x_{k}\right)$ and therefore, by the three-spheres inequality (4.19),

$$
\|u\|_{L^{2}\left(B_{r_{1}}\left(x_{k+1}\right)\right)}+\varepsilon \leq Q\left(\|u\|_{L^{2}\left(B_{r_{1}}\left(x_{k}\right)\right)}+\varepsilon\right)^{\alpha}\left(E_{0}+\varepsilon\right)^{1-\alpha},
$$

for $k=0, \ldots, N-1$, where $Q \geq 1$ and $\alpha, 0<\alpha<1$, only depend on $K, L$ and $\kappa$.

Denoting

$$
m_{k}=\frac{\|u\|_{L^{2}\left(B_{r_{1}}\left(x_{k}\right)\right)}+\varepsilon}{E_{0}+\varepsilon}
$$

we then have

$$
m_{k+1} \leq Q m_{k}^{\alpha}, \quad \text { for } k=0, \ldots, N-1,
$$




$$
m_{N} \leq \widetilde{Q} m_{0}^{\delta}
$$

where $\widetilde{Q}=Q^{1+\alpha+\ldots+\alpha^{N-1}}$ and $\delta=\alpha^{N}$. Hence we have obtained

$$
\|u\|_{L^{2}\left(B_{r_{1}}(y)\right)} \leq \widetilde{Q}\left(\|u\|_{L^{2}\left(B_{r_{1}}\left(x_{0}\right)\right)}+\varepsilon\right)^{\delta}\left(E_{0}+\varepsilon\right)^{1-\delta} .
$$

Now, $1+\alpha+\ldots+\alpha^{N-1} \leq \frac{1}{1-\alpha}$. Since $B_{r_{1}}\left(x_{0}\right), \ldots, B_{r_{1}}\left(x_{N-1}\right)$ are pairwise disjoint, we have that $N \leq \frac{|\Omega|}{\omega_{n} r_{1}^{n}} \leq \frac{C_{2}|\Omega|}{h^{n}}$, with $C_{2}$ only depending on $K$. Hence, recalling that $Q \geq 1$, we compute

$$
\widetilde{Q} \leq Q^{\frac{1}{1-\alpha}}
$$

$$
\delta \geq \alpha^{\frac{C_{2}|\Omega|}{h^{n}}}
$$

Let us tessellate $\mathbb{R}^{n}$ with internally non-overlapping closed cubes of side $l=\frac{2 r_{1}}{\sqrt{n}}$ and let $Q_{j}, j=1, \ldots, J$, be those cubes which intersect $G$. Clearly, any such cube is contained in a ball of radius $r_{1}$ and center $w_{j} \in G^{r_{1}}$ and $J \leq \frac{n^{\frac{n}{2}}|\Omega|}{2^{n} r_{1}^{n}}$. Therefore, from (5.13), we have

$$
\int_{G} u^{2} \leq \sum_{j=1}^{J} \int_{Q_{j}} u^{2} \leq \sum_{j=1}^{J} \int_{B_{r_{1}}\left(w_{j}\right)} u^{2} \leq J \widetilde{Q}^{2} \rho_{0}^{n}\left(\|u\|_{L^{2}\left(B_{r_{1}}\left(x_{0}\right)\right)}+\varepsilon\right)^{2 \delta}\left(E_{0}+\varepsilon\right)^{2(1-\delta)} .
$$

Hence the thesis immediately follows.

Remark 5.2 It is important at this stage to emphasize that the above Theorem 5.1 on propagation of smallness in the interior enables us to generalize the three-spheres inequality of Theorem 4.4, by removing the limitations on the radii that were present there. It should also be mentioned however that by this approach it does not seem possible to obtain optimal estimations of the constants $C, \alpha$ appearing in the inequality (1.42). This optimization problem for such a general version of the three-spheres inequality still mantains some unanswered aspects.

Proof of Theorem 1.10 It follows by applying Theorem 5.1, with $B_{r_{0}}\left(x_{0}\right)=B_{r_{1}}, G=B_{r_{2}}$, $\Omega=B_{r_{3}}$.

The dependence of $C, \alpha$ on the quantities stated in Theorem 1.10 is straightforward, although somewhat lengthy.

Theorem 5.3 (Global propagation of smallness) Let $\Omega$ be a bounded connected open set in $\mathbb{R}^{n}$ with boundary $\partial \Omega$ of Lipschitz class with constants $\rho_{0}, M_{0}$. Let $u \in H^{1}(\Omega)$ be a solution to the equation

$$
\operatorname{div}(A \nabla u)+c u=f+\operatorname{div} F, \quad \text { in } \Omega,
$$

where $f$ and $F$ satisfy (1.29). Let $B_{r_{0}}\left(x_{0}\right) \subset \Omega$ and let us assume that

$$
\|u\|_{L^{2}\left(B_{r_{0}}\left(x_{0}\right)\right)} \leq \eta
$$




$$
\|u\|_{H^{1}(\Omega)} \leq E
$$

for given $\eta>0, E>0$. We have

$$
\|u\|_{L^{2}(\Omega)} \leq(E+\varepsilon) \omega\left(\frac{\eta+\varepsilon}{E+\varepsilon}\right),
$$

where

$$
\omega(t) \leq \frac{C}{\left(\log \frac{1}{t}\right)^{\mu}}, \quad \text { for } t<1 .
$$

where $C>0$ and $\mu, 0<\mu<1$, only depend on $K, L, \kappa, M_{0}, \frac{r_{0}}{\rho_{0}}$ and $\frac{|\Omega|}{\rho_{0}^{n}}$.

Remark 5.4 As it will be evident from the proof, rather than the bound (5.19) we shall actually use a weaker one. In fact we might replace (5.19) by the assumption that there exists a $p>2$ such that

$$
\|u\|_{L^{p}(\Omega)} \leq E .
$$

In this case the constants $C, \mu$ would also depend on such an exponent $p>2$. We have chosen to formulate Theorem 5.3 (and also Theorem [1.9) in terms of an a-priori $H^{1}$-bound because in many applied settings such a bound has a clearer physical interpretation.

We premise the following proposition.

Proposition 5.5 Let $\Omega$ be a bounded connected open set in $\mathbb{R}^{n}$ with boundary $\partial \Omega$ of Lipschitz class with constants $\rho_{0}, M_{0}$. There exists $h_{0}>0$, only depending on $\rho_{0}, M_{0}$, such that $\Omega_{h}$ is connected for every $h<h_{0}$.

In order to prove the above proposition, let us introduce the following lemma. We recall our assumptions. $\Omega$ is a bounded connected open set in $\mathbb{R}^{n}$ with boundary $\partial \Omega$ of Lipschitz class with constants $\rho_{0}, M_{0}$. Let $P \in \partial \Omega$. According to Definition 1.1, up to a rigid motion, we have $P=0$ and

$$
\Omega \cap \Gamma_{\frac{\rho_{0}}{M_{0}}, \rho_{0}}(P)=\left\{x=\left(x^{\prime}, x_{n}\right) \in \Gamma_{\frac{\rho_{0}}{M_{0}}, \rho_{0}} \quad \mid \quad x_{n}>Z\left(x^{\prime}\right)\right\},
$$

where $Z$ is a Lipschitz function on $B_{\frac{\rho_{0}}{M_{0}}}^{{ }_{M}} \subset \mathbb{R}^{n-1}$ satisfying

$$
\begin{gathered}
Z(0)=0, \\
\|Z\|_{C^{0,1}\left(B_{\rho_{0}^{\prime}}^{\frac{\rho_{0}}{M_{0}}}\right)} \leq M_{0} \rho_{0} .
\end{gathered}
$$

We denote

$$
\begin{gathered}
d(x)=\operatorname{dist}(x, \partial \Omega), \quad x \in \Omega, \\
\widetilde{d}(x)=x_{n}-Z\left(x^{\prime}\right), \quad x \in \Omega \cap \Gamma \frac{\rho_{0}}{M_{0}}, \rho_{0},
\end{gathered}
$$

and also

$$
d_{0}=\frac{\rho_{0}}{2 M_{0}} .
$$


Lemma 5.6 Under the above stated assumptions, if we assume

$$
x \in \Gamma_{\frac{\rho_{0}}{2 M_{0}}, \frac{\rho_{0}}{2}} \cap \Omega, \quad d(x) \leq d_{0},
$$

then we have

$$
d(x) \leq \widetilde{d}(x) \leq\left(1+\sqrt{1+M_{0}^{2}}\right) d(x)
$$

Proof Let $x \in \Gamma_{\frac{\rho_{0}}{2 M_{0}}}, \frac{\rho_{0}}{2} \cap \Omega$ be such that $d(x) \leq d_{0}$. Let $z \in \partial \Omega$ be such that $|z-x|=d(x)$. Recalling that $M_{0} \geq 1$, we have that

$$
\begin{gathered}
\left|z^{\prime}\right| \leq\left|z^{\prime}-x^{\prime}\right|+\left|x^{\prime}\right|<d(x)+\frac{\rho_{0}}{2 M_{0}} \leq \frac{\rho_{0}}{M_{0}}, \\
\left|z_{n}\right| \leq\left|z_{n}-x_{n}\right|+\left|x_{n}\right|<d(x)+\frac{\rho_{0}}{2} \leq \frac{\rho_{0}}{2 M_{0}}+\frac{\rho_{0}}{2} \leq \rho_{0} .
\end{gathered}
$$

Therefore $z \in \Gamma_{\frac{\rho_{0}}{M_{0}}, \rho_{0}} \cap \partial \Omega$, so that $z=\left(z^{\prime}, Z\left(z^{\prime}\right)\right)$.

Let $y=\left(x^{\prime}, Z\left(x^{\prime}\right)\right)$. We have that

$$
\begin{gathered}
d(x)=|x-z| \leq|x-y|=\widetilde{d}(x), \\
\widetilde{d}(x)=|x-y| \leq|x-z|+|z-y|=|x-z|+\left(\left|x^{\prime}-z^{\prime}\right|^{2}+\left|Z\left(x^{\prime}\right)-Z\left(z^{\prime}\right)\right|^{2}\right)^{\frac{1}{2}} \leq \\
\leq|x-z|+\sqrt{1+M_{0}^{2}}\left|x^{\prime}-z^{\prime}\right| \leq\left(1+\sqrt{1+M_{0}^{2}}\right) d(x) .
\end{gathered}
$$

Proof of Proposition 5.5 Let $h_{0}=\frac{\rho_{0}}{4 M_{0}\left(1+\sqrt{1+M_{0}^{2}}\right)}$. We trivially have that $h_{0}<\frac{\rho_{0}}{8 M_{0}}$. Let $h<h_{0}$. Let us first prove that $\overline{\Omega_{h}}$ is connected. Given $x, y \in \overline{\Omega_{h}}$, let $\gamma$ be a path in $\Omega$ connecting $x$ and $y$. If $\gamma$ is not contained in $\overline{\Omega_{h}}$, let us modify $\gamma$ to a new path $\widetilde{\gamma}$ connecting $x$ and $y$ and contained in $\overline{\Omega_{h}}$. Let $C=\{\gamma(t) \mid d(\gamma(t)) \leq h\}$. For every $z \in C$, there exists $\xi_{z} \in \partial \Omega$ such that $\left|z-\xi_{z}\right| \leq h$ so that $z \in \Gamma_{\frac{\rho_{0}}{8 M_{0}}}, \frac{\rho_{0}}{8}\left(\xi_{z}\right)$, where $\Gamma_{\frac{\rho_{0}}{8 M_{0}},}, \frac{\rho_{0}}{8}\left(\xi_{z}\right)$ is defined according to the Definition 1.1. Therefore the cylinders $\Gamma_{\frac{\rho_{0}}{8 M_{0}},}, \frac{\rho_{0}}{8}\left(\xi_{z}\right)$ provide an open covering of $C$ and, since $C$ is compact, there exist $z_{1}, \ldots, z_{N}$ such that $C \subset \cup_{i=1}^{N} \Gamma \frac{\rho_{0}}{8 M_{0}}, \frac{\rho_{0}}{8}\left(\xi_{z_{i}}\right)$. Let us denote for simplicity $\xi_{i}=\xi_{z_{i}}, \Gamma^{i}=\Gamma_{\frac{\rho_{0}}{8 M_{0}}, \frac{\rho_{0}}{8}}\left(\xi_{i}\right)$. Note that each such cylinder is possibly oriented with respect to a different coordinate system. For every point $\gamma(t) \in C \cap \overline{\Gamma^{1}}$ let us replace $\gamma(t)$ with $\widetilde{\gamma}(t)=\left(\gamma^{\prime}(t), Z\left(\gamma^{\prime}(t)\right)+\left(1+\sqrt{1+M_{0}^{2}}\right) h\right)$. We can apply Lemma [5.6] to $\gamma(t)$, obtaining that $\gamma_{n}(t)-Z\left(\gamma^{\prime}(t)\right)=\widetilde{d}(\gamma(t)) \leq\left(1+\sqrt{1+M_{0}^{2}}\right) h$. Recalling that $M_{0} \geq 1$, we have that

$$
\begin{gathered}
\left|\widetilde{\gamma}^{\prime}(t)-\left(\xi_{1}\right)^{\prime}\right|=\left|\gamma^{\prime}(t)-\left(\xi_{1}\right)^{\prime}\right| \leq \frac{\rho_{0}}{8 M_{0}}<\frac{\rho_{0}}{2 M_{0}} \\
\left|\widetilde{\gamma}_{n}(t)-\xi_{n}^{1}\right| \leq\left|\widetilde{\gamma}_{n}(t)-\gamma_{n}(t)\right|+\left|\gamma_{n}(t)-\xi_{n}^{1}\right| \leq\left(1+\sqrt{1+M_{0}^{2}}\right) h+\frac{\rho_{0}}{8}<\frac{\rho_{0}}{2} .
\end{gathered}
$$


Therefore $\widetilde{\gamma}(t) \in \Gamma_{\frac{\rho_{0}}{2 M_{0}}, \frac{\rho_{0}}{2}}\left(\xi^{1}\right) \cap \Omega$ and $d(\widetilde{\gamma}(t)) \leq \mid \widetilde{\gamma}(t)-\left(\gamma^{\prime}(t), Z\left(\gamma^{\prime}(t)\right) \mid \leq\left(1+\sqrt{1+M_{0}^{2}}\right) h<\right.$ $d_{0}$. We can therefore apply Lemma 5.6 to $\widetilde{\gamma}(t)$ and, by (5.27),

$$
d(\widetilde{\gamma}(t)) \geq \frac{\widetilde{d}(\widetilde{\gamma}(t))}{1+\sqrt{1+M_{0}^{2}}}=h .
$$

The connected components of $\gamma^{-1}\left(C \cap \overline{\Gamma^{1}}\right)$ are closed intervals $I_{\alpha}$. In order to glue together $\gamma$ and $\widetilde{\gamma}$, let us add, for each endpoint $t$ of any $I_{\alpha}$ and for any $t \in \gamma^{-1}\left(C \cap \partial \Gamma^{1}\right)$, the closed segment joining $\left(\gamma^{\prime}(t), \gamma_{n}(t)\right)$ and $\left(\gamma^{\prime}(t), Z\left(\gamma^{\prime}(t)\right)+\left(1+\sqrt{1+M_{0}^{2}}\right) h\right)$. Now we repeat the above arguments to the path so modified and to the cylinder $\Gamma^{2}$, and so on for a finite number of steps.

We can now conclude proving that $\Omega_{h}$ is connected for every $h<h_{0}$. Let $x, y \in \Omega_{h}$ and let $h^{\prime}$ such that $h<h^{\prime}<\min \left\{d(x), d(y), h_{0}\right\}$. We have just shown that $\bar{\Omega}_{h^{\prime}}$ is path connected, hence there exists a path in $\overline{\Omega_{h^{\prime}}} \subset \Omega_{h}$ joining $x$ and $y$.

Lemma 5.7 Let $\Omega$ be a bounded connected open set in $\mathbb{R}^{n}$ with boundary $\partial \Omega$ of Lipschitz class with constants $\rho_{0}, M_{0}$. There exists a constant $C>0$, only depending on $M_{0}$, such that

$$
\mu\left(\Omega \backslash \Omega_{h}\right) \leq C|\Omega| \frac{h}{\rho_{0}} .
$$

Proof We refer to [11, (A.3)] for a detailed proof. In fact this result was proved there under stronger regularity assumptions, but the same arguments apply also under our Lipschitz regularity assumption.

Proof of Theorem 5.3 Let $h_{1}=\min \left\{\frac{h_{0}}{2}, \frac{r_{0}}{2}, 2 C_{0} \rho_{0}\right\}$, where $h_{0}=\frac{\rho_{0}}{4 M_{0}\left(1+\sqrt{1+M_{0}^{2}}\right)}$ has been introduced in Proposition 5.5. We have that $\Omega_{h_{1}}$ is connected, it contains $B_{\frac{r_{0}}{2}}\left(x_{0}\right)$ and also we can apply Theorem 5.1 , with $G=\Omega_{h_{1}}$. That is

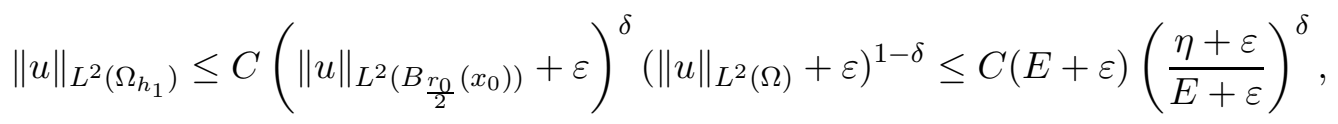

where $C>0$ and $\delta, 0<\delta<1$, only depend on $K, L, \kappa, M_{0}, \frac{\rho_{0}}{r_{0}}$, and $\frac{|\Omega|}{\rho_{0}^{n}}$.

Let $r \in\left(0, h_{1}\right)$. Given any point $x \in \Omega_{r} \backslash \Omega_{h_{1}}$, that is such that $r<d(x) \leq h_{1}$, let $\xi \in \partial \Omega$ be such that $d=d(x)=|\xi-x|$.

According to the Definition 1.1, up to a rigid motion, $\Omega \cap \Gamma_{\frac{\rho_{0}}{M_{0}}, \rho_{0}}(\xi)=\left\{y=\left(y^{\prime}, y_{n}\right) \in\right.$ $\left.\Gamma \frac{\rho_{0}}{M_{0}}, \rho_{0} \mid y_{n}>Z\left(y^{\prime}\right)\right\}$, with $Z$ a Lipschitz function on $B_{\frac{\rho_{0}}{M_{0}}}^{\prime} \subset \mathbb{R}^{n-1}$ satisfying $Z(0)=0$, $\|Z\|_{C^{0,1}} \leq M_{0} \rho_{0}$.

By (5.27), we have that $r<d \leq \widetilde{d}(x)=x_{n}-Z\left(x^{\prime}\right) \leq\left(1+\sqrt{1+M_{0}^{2}}\right) d$. Let $w=$ $\left(x^{\prime}, Z\left(x^{\prime}\right)\right) \in \partial \Omega$. We have that $\left|x^{\prime}\right| \leq d,\left|Z\left(x^{\prime}\right)\right| \leq M_{0} d$.

Let us translate the origin of the coordinate system from $\xi$ to $w$.

Let $t_{0}=\frac{\sqrt{1+M_{0}^{2}}}{1+\sqrt{1+M_{0}^{2}}}\left(\rho_{0}-M_{0} h_{1}\right), s_{0}=\frac{1}{4}\left(\frac{\rho_{0}-M_{0} h_{1}}{1+\sqrt{1+M_{0}^{2}}}-h_{1}\right)$ and $y_{0}=\left(0, t_{0}\right)$. We have that

$$
4 s_{0}+h_{1}=\frac{t_{0}}{\sqrt{1+M_{0}^{2}}}
$$




$$
\begin{gathered}
t_{0}+4 s_{0}+h_{1} \leq \rho_{0}-M_{0} d, \\
4 s_{0} \leq \frac{\rho_{0}}{M_{0}}-d,
\end{gathered}
$$

so that

$$
B_{4 s_{0}}\left(y_{0}\right) \subset \Omega_{h_{1}} \cap \Gamma_{\frac{\rho_{0}}{M_{0}}-d, \rho_{0}-M_{0} d}(w) \subset \Omega_{h_{1}} \cap \Gamma_{\frac{\rho_{0}}{M_{0}}, \rho_{0}}(\xi) .
$$

Let $C_{w}=\left\{y=\left(y^{\prime}, y_{n}\right)\left|y_{n}>\widetilde{M}\right| y^{\prime} \mid\right\}$ be the open cone with vertex at $w$ and tangent to the ball $B_{s_{0}}\left(y_{0}\right)$, that is $\widetilde{M}=\frac{1}{\tan \vartheta}$, with $\sin \vartheta=\frac{s_{0}}{t_{0}}$. Let us denote $s=\sin \vartheta, q=\frac{1-s}{1+s}$. Let $s_{1}=q s_{0}, t_{1}=q t_{0}$ and $y_{1}=\left(0, t_{1}\right)$. Then the ball $B_{s_{1}}\left(y_{1}\right)$ is tangent to the cone $C_{w}$ and to the ball $B_{s_{0}}\left(y_{0}\right)$. By induction, setting $s_{k}=q s_{k-1}=q^{k} s_{0}, t_{k}=q t_{k-1}=q^{k} t_{0}, y_{k}=\left(0, t_{k}\right)$, for $k \geq 2$, we have that the ball $B_{s_{k}}\left(y_{k}\right)$ is tangent to the cone $C_{w}$ and to the ball $B_{s_{k-1}}\left(y_{k-1}\right)$. Let

$$
m_{k}=\frac{\|u\|_{L^{2}\left(B_{s_{k}}\left(y_{k}\right)\right)}+\varepsilon}{E+\varepsilon} .
$$

By applying (1.42) to the balls of center $y_{k}$ and radii $s_{k}, 3 s_{k}, 4 s_{k}$, and noticing that $B_{s_{k+1}}\left(y_{k+1}\right) \subset B_{3 s_{k}}\left(y_{k}\right)$, we have

$$
\begin{gathered}
m_{k+1} \leq C m_{k}^{\alpha}, \quad \text { for every } k=0,1, \ldots, \\
m_{N} \leq C^{1+\alpha+\ldots+\alpha^{N-1}} m_{0}^{\alpha^{N}}, \quad \text { for every } N=1,2, \ldots,
\end{gathered}
$$

where $C>0$ and $\alpha, 0<\alpha<1$, only depend on $K, L$ and $\kappa$. By (5.29) we have

$$
m_{0} \leq C\left(\frac{\eta+\varepsilon}{E+\varepsilon}\right)^{\delta}
$$

so that

$$
m_{N} \leq C\left(\left(\frac{\eta+\varepsilon}{E+\varepsilon}\right)^{\delta}\right)^{\alpha^{N}}
$$

where $\alpha \in(0,1)$ only depends on $K, L$ and $\kappa$, whereas $C>0$ and $\delta, 0<\delta<1$, only depend on $K, L, \kappa, M_{0}, \frac{\rho_{0}}{r_{0}}$ and $\frac{|\Omega|}{\rho_{0}^{n}}$.

In the new coordinate system centered at $w, x=(0, \widetilde{d}(x))$ and, by (5.27) and by the choice of $t_{0}, r<\widetilde{d}(x) \leq\left(1+\sqrt{1+M_{0}^{2}}\right) d \leq\left(1+\sqrt{1+M_{0}^{2}}\right) h_{1}<t_{0}$. Since $x \notin \Omega_{h_{1}}$, we have that $x \notin B_{s_{0}}\left(y_{0}\right) \cup B_{s_{1}}\left(y_{1}\right) \subset B_{3 s_{0}}\left(y_{0}\right) \subset \Omega_{h_{1}}$. Hence there exists $N \in \mathbb{N}, N \geq 2$, such that $x \in \overline{B_{s_{N}}\left(y_{N}\right)}, x \notin B_{s_{N-1}}\left(y_{N-1}\right)$. It follows that $\widetilde{d}(x)<t_{N-1}$ so that $q^{N-1}>\frac{r}{t_{0}}$.

By (1.42) and by (5.33), we have

$$
\frac{\|u\|_{L^{2}\left(B_{3 s_{N}}\left(y_{N}\right)\right)}}{E+\varepsilon} \leq C m_{N}^{\alpha} \leq C\left(\frac{\eta+\varepsilon}{E+\varepsilon}\right)^{\gamma \alpha^{N-1}}
$$

where $C>0$ and $\gamma=\alpha \delta$ only depend on $K, L, \kappa, M_{0}, \frac{\rho_{0}}{r_{0}}$ and $\frac{|\Omega|}{\rho_{0}^{n}}$. Since $\alpha^{N-1}=q^{(N-1) \frac{\log \alpha}{\log q}}>$ $\left(\frac{r}{t_{0}}\right)^{\frac{\log \alpha}{\log q}}$, and noticing that $\frac{\eta+\varepsilon}{E+\varepsilon}<1$, we have

$$
\|u\|_{L^{2}\left(B_{3 s_{N}}\left(y_{N}\right)\right)} \leq C(E+\varepsilon)\left(\frac{\eta+\varepsilon}{E+\varepsilon}\right)^{\gamma\left(\frac{r}{t_{0}}\right)^{D}},
$$


with $D=\frac{\log \alpha}{\log q}$ and $C>0$ and $\gamma \in(0,1)$ only depending on $K, L, \kappa, M_{0}, \frac{\rho_{0}}{r_{0}}$ and $\frac{|\Omega|}{\rho_{0}^{n}}$. Moreover $s_{N}=q^{N} s_{0}>\frac{r q s_{0}}{t_{0}}$. Since $B_{2 s_{N}}(x) \subset B_{3 s_{N}}\left(y_{N}\right)$, we have that

$$
\|u\|_{L^{2}\left(B_{2 s_{N}}(x)\right)} \leq C(E+\varepsilon)\left(\frac{\eta+\varepsilon}{E+\varepsilon}\right)^{\gamma\left(\frac{r}{t_{0}}\right)^{D}}
$$

with $C>0$ and $\gamma \in(0,1)$ only depending on $K, L, \kappa, M_{0}, \frac{\rho_{0}}{r_{0}}$ and $\frac{|\Omega|}{\rho_{0}^{n}}$. Let us tessellate $\mathbb{R}^{n}$ with closed cubes of side $l=\frac{2 r q s_{0}}{\sqrt{n} t_{0}}$ and let $Q_{j}, j=1, \ldots, J$, be those cubes which intersect $\Omega_{r} \backslash \Omega_{h_{1}}$. Clearly, any such cube is contained in a ball of radius $\frac{r q s_{0}}{t_{0}}$ and center $w_{j}$ such that $\left|w_{j}-x_{j}\right| \leq \frac{r q s_{0}}{t_{0}}$, for some $x_{j} \in \Omega_{r} \backslash \Omega_{h_{1}}$. By the above arguments, there exists $N \in \mathbb{N}$, $N \geq 2$, such that (5.36) holds for $x=x_{j}$, with $s_{N}>\frac{r q s_{0}}{t_{0}}$. Therefore $B_{s_{N}}\left(w_{j}\right) \subset B_{2 s_{N}}\left(x_{j}\right)$ and, by (5.36) and by the trivial estimate $J \leq \frac{n^{\frac{n}{2}} t_{0}^{n}|\Omega|}{\left(2 q r s_{0}\right)^{n}}$, we have that

$$
\int_{\Omega_{r} \backslash \Omega_{h_{1}}} u^{2} \leq \sum_{j=1}^{J} \int_{Q_{j}} u^{2} \leq \sum_{j=1}^{J} \int_{B s_{N}\left(w_{j}\right)} u^{2} \leq C \rho_{0}^{n}(E+\varepsilon)^{2}\left(\frac{r}{t_{0}}\right)^{-n}\left(\frac{\eta+\varepsilon}{E+\varepsilon}\right)^{2 \gamma\left(\frac{r}{t_{0}}\right)^{D}},
$$

where $C>0$ and $\gamma \in(0,1)$ only depend on $K, L, \kappa, M_{0}, \frac{\rho_{0}}{r_{0}}$ and $\frac{|\Omega|}{\rho_{0}^{n}}$. By (5.29) and by (5.37), we arrive at

$$
\|u\|_{L^{2}\left(\Omega_{r}\right)} \leq C(E+\varepsilon)\left(\frac{r}{t_{0}}\right)^{-\frac{n}{2}}\left(\frac{\eta+\varepsilon}{E+\varepsilon}\right)^{\gamma\left(\frac{r}{t_{0}}\right)^{D}}
$$

with $C>0$ and $\gamma \in(0,1)$ only depending on $K, L, \kappa, M_{0}, \frac{\rho_{0}}{r_{0}}$ and $\frac{|\Omega|}{\rho_{0}^{n}}$. Let us choose $p=\frac{2 n}{n-2}$, for $n>2$, whereas for $n=2$, let us choose as $p$ any number satisfying $p>2$. From Hölder inequality, Sobolev embedding theorem and by Lemma 5.7, we deduce

$$
\begin{aligned}
&\|u\|_{L^{2}\left(\Omega \backslash \Omega_{r}\right)} \leq\left(\frac{\left|\Omega \backslash \Omega_{r}\right|}{\rho_{0}^{n}}\right)^{\frac{1}{2}-\frac{1}{p}}\|u\|_{L^{p}(\Omega)} \leq \\
& \leq C\left(\frac{\left|\Omega \backslash \Omega_{r}\right|}{\rho_{0}^{n}}\right)^{\frac{1}{2}-\frac{1}{p}} \rho_{0}\|\nabla u\|_{L^{2}(\Omega)} \leq C E\left(\frac{r}{\rho_{0}}\right)^{\frac{1}{2}-\frac{1}{p}}
\end{aligned}
$$

with $C$ only depending on $M_{0}$ and $\frac{|\Omega|}{\rho_{0}^{n}}$. Recall that $t_{0}<\rho_{0}$, therefore we may replace $\rho_{0}$ with $t_{0}$ in (5.39). By (5.38) and (5.39), we have that for every $r, 0<r<h_{1}$,

$$
\|u\|_{L^{2}(\Omega)} \leq C(E+\varepsilon)\left(\frac{r}{t_{0}}\right)^{-\frac{n}{2}}\left(\left(\frac{\eta+\varepsilon}{E+\varepsilon}\right)^{\gamma\left(\frac{r}{t_{0}}\right)^{D}}+\left(\frac{r}{t_{0}}\right)^{\frac{1}{2}-\frac{1}{p}}\right)
$$

with $C>0$ and $\gamma \in(0,1)$ only depending on $K, L, \kappa, M_{0}, \frac{\rho_{0}}{r_{0}}$ and $\frac{|\Omega|}{\rho_{0}^{n}}$. Setting

$$
\tau=\left(\frac{r}{t_{0}}\right)^{D}, \quad \tau_{0}=\left(\frac{h_{1}}{t_{0}}\right)^{D}
$$




$$
\vartheta=\frac{1}{D}\left(\frac{1}{2}-\frac{1}{p}\right), \quad \sigma=\frac{n}{2 D}, \quad \zeta=\left(\frac{\eta+\varepsilon}{E+\varepsilon}\right)^{\gamma}
$$

we obtain

$$
\|u\|_{L^{2}(\Omega)} \leq C(E+\varepsilon)\left(\tau^{\vartheta}+\tau^{-\sigma} \zeta^{\tau}\right), \quad \text { for every } \tau, 0<\tau \leq \tau_{0}
$$

with $C>0$ and $\gamma \in(0,1)$ only depending on $K, L, \kappa, M_{0}, \frac{\rho_{0}}{r_{0}}$ and $\frac{|\Omega|}{\rho_{0}^{n}}$. Denoting

$$
\Phi(\tau)=\tau^{\vartheta}+\tau^{-\sigma} \zeta^{\tau}
$$

let us estimate from above $\inf _{0<\tau<\tau_{0}} \Phi(\tau)$. To this aim, it is convenient to introduce a new parameter $l$, related to $\tau$ by

$$
\tau=\left(\frac{1}{\log \frac{1}{\zeta}}\right)^{l}
$$

We compute

$$
\Phi(\tau)=\left(\frac{1}{\log \frac{1}{\zeta}}\right)^{l \vartheta}+\left(\frac{1}{\log \frac{1}{\zeta}}\right)^{-l \sigma} \exp \left\{-\left(\log \frac{1}{\zeta}\right) \frac{1}{\left(\log \frac{1}{\zeta}\right)^{l}}\right\} .
$$

Let us temporarily assume $l<1$, and let us use the inequality $e^{-s}<1 / s$ with

$$
s=\frac{1}{\left(\log \frac{1}{\zeta}\right)^{l-1}} .
$$

We obtain

$$
\Phi(\tau) \leq\left(\frac{1}{\log \frac{1}{\zeta}}\right)^{l \vartheta}+\left(\frac{1}{\log \frac{1}{\zeta}}\right)^{1-l(1+\sigma)} .
$$

Let us choose $l=\frac{1}{1+\vartheta+\sigma}$, so that $l \vartheta=1-l(1+\sigma)=\frac{\vartheta}{1+\vartheta+\sigma}$, and $0<l<1$. Denoting

$$
\mu=\min \{l \vartheta, 1-l(1+\sigma)\}=\frac{\vartheta}{1+\vartheta+\sigma},
$$

we have

$$
\Phi(\tau) \leq 2\left(\frac{1}{\log \frac{1}{\zeta}}\right)^{\mu}
$$

Now, if $\left(\frac{1}{\log \frac{1}{\zeta}}\right)^{l} \leq \tau_{0}$, then $\min _{0<\tau \leq \tau_{0}} \Phi(\tau) \leq 2\left(\frac{1}{\log \frac{1}{\zeta}}\right)^{\mu}$. Otherwise, if $\left(\frac{1}{\log \frac{1}{\zeta}}\right)^{l}>\tau_{0}$, then $\min _{0<\tau \leq \tau_{0}} \Phi(\tau) \leq \Phi\left(\tau_{0}\right) \leq C$, with $C>0$ only depending on $K, L, \kappa, M_{0}$ and $\frac{r_{0}}{\rho_{0}}$.

Furthermore, $\left(\frac{1}{\log \frac{1}{\zeta}}\right)^{\mu}>\tau_{0}^{\frac{\mu}{l}}$, and hence $\min _{0<\tau \leq \tau_{0}} \Phi(\tau) \leq \frac{C}{\tau_{0}^{\frac{\mu}{l}}}\left(\frac{1}{\log \frac{1}{\zeta}}\right)^{\mu}$. By (5.41) and recalling the definition of $\zeta$, we arrive at

$$
\|u\|_{L^{2}(\Omega)} \leq C(E+\varepsilon)\left(\frac{1}{-\gamma \log \frac{\eta+\varepsilon}{E+\varepsilon}}\right)^{\mu}
$$


where $C>0$ and $\mu, 0<\mu<1$, only depend on $K, L, \kappa, M_{0}, \frac{r_{0}}{\rho_{0}}$ and $\frac{|\Omega|}{\rho_{0}^{n}}$. Therefore, with a possibly new choice of $C$, we obtain

$$
\|u\|_{L^{2}(\Omega)} \leq C(E+\varepsilon)\left(\frac{1}{\log \frac{E+\varepsilon}{\eta+\varepsilon}}\right)^{\mu},
$$

where $C>0$ and $\mu, 0<\mu<1$, only depend on $K, L, \kappa, M_{0}, \frac{r_{0}}{\rho_{0}}$ and $\frac{|\Omega|}{\rho_{0}^{n}}$.

\section{Proofs of the stability results for the Cauchy problem}

In order to reduce the problem of continuation from Cauchy data to a problem of continuation from an open set, we shall introduce an augmented domain as follows.

We recall that $\Sigma$ is an open Lipschitz portion of $\partial \Omega$ with constants $\rho_{0}, M_{0}$ according to Definition 1.4 and it has size at least $\rho_{1}>0$ according to Definition 1.5 .

We denote $\eta:[0,+\infty) \rightarrow[0,1]$ as follows

$$
\eta(t)= \begin{cases}1, & 0 \leq t \leq \frac{1}{4}, \\ 4\left(\frac{1}{2}-t\right), & \frac{1}{4} \leq t \leq \frac{1}{2} \\ 0, & t \geq \frac{1}{2} .\end{cases}
$$

Let $P \in \Sigma$ the point described in Definition 1.5 and let $Z: B_{\frac{\rho_{0}}{M_{0}}}^{\prime} \rightarrow \mathbb{R}$ be the Lipschitz function appearing in (1.16) for the local representation of $\Omega$ near $P$. According to (1.16) we have

$$
\Omega \cap \Gamma_{\frac{\rho_{1}}{M_{0}}, \rho_{1}}(P)=\left\{x=\left(x^{\prime}, x_{n}\right) \in \Gamma_{\frac{\rho_{1}}{M_{0}}, \rho_{1}} \quad \mid \quad x_{n}>Z\left(x^{\prime}\right)\right\} .
$$

Let us denote

$$
Z^{-}\left(x^{\prime}\right)=Z\left(x^{\prime}\right)-\frac{\rho_{1}}{2} \eta\left(\frac{M_{0}\left|x^{\prime}\right|}{\rho_{1}}\right), \quad \text { for every } x^{\prime} \in B_{\frac{\rho_{0}}{M_{0}}} .
$$

Observe that

$$
\begin{gathered}
\left|Z^{-}\left(x^{\prime}\right)\right| \leq\left|Z\left(x^{\prime}\right)\right|+\frac{\rho_{1}}{2}, \quad \text { for every } x^{\prime} \in B_{\frac{\rho_{0}}{M_{0}}}^{\prime}, \\
\left|\nabla_{x^{\prime}} Z^{-}\left(x^{\prime}\right)\right| \leq\left|\nabla_{x^{\prime}} Z\left(x^{\prime}\right)\right|+2 M_{0}, \quad \text { for every } x^{\prime} \in B_{\frac{\rho_{0}}{M_{0}}}^{\prime},
\end{gathered}
$$

and therefore, since $M_{0} \geq 1$,

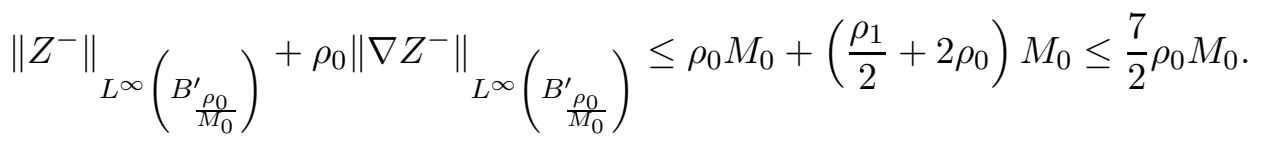

Next, we denote

$$
\mathcal{A}=\left\{x=\left(x^{\prime}, x_{n}\right) \in \Gamma_{\frac{\rho_{0}}{M_{0}}, \rho_{0}} \quad \mid \quad Z^{-}\left(x^{\prime}\right)<x_{n}<Z\left(x^{\prime}\right)\right\},
$$




$$
\begin{gathered}
\Sigma_{0}=\left\{x=\left(x^{\prime}, x_{n}\right) \in \Gamma_{\frac{\rho_{0}}{M_{0}}, \rho_{0}} \quad|\quad| x^{\prime} \mid<\frac{\rho_{1}}{2 M_{0}}, x_{n}=Z\left(x^{\prime}\right)\right\}, \\
\widetilde{\Omega}=\Omega \cup \Sigma_{0} \cup \mathcal{A} .
\end{gathered}
$$

It is a straightforward matter to verify that

i) if $\Omega$ has Lipschitz boundary with constants $\rho_{0}, M_{0}$, then $\widetilde{\Omega}$ has Lipschitz boundary with constants $\frac{\rho_{0}}{2}, \frac{7}{2} M_{0}$.

ii) using the coordinates employed in the construction we have

$$
\widetilde{\Omega} \supset \Gamma_{\frac{\rho_{1}}{4 M_{0}}, \frac{\rho_{1}}{4}} .
$$

iii) if we denote, for any $r>0$,

$$
\begin{gathered}
C_{r}^{-}=\left\{x=\left(x^{\prime}, x_{n}\right) \in \mathbb{R}^{n}\left|-r<x_{n}<-M_{0}\right| x^{\prime} \mid\right\}, \\
C_{r}^{+}=\left\{x=\left(x^{\prime}, x_{n}\right) \in \mathbb{R}^{n}\left|M_{0}\right| x^{\prime} \mid<x_{n}<r\right\},
\end{gathered}
$$

we also have

$$
\mathcal{A} \supset C_{\frac{\rho_{1}}{4}}^{-}
$$

iv) if we denote

$$
\begin{gathered}
r_{0}=\frac{\rho_{1}\left(\sqrt{1+M_{0}^{2}}-1\right)}{8 M_{0}^{2}}=\frac{\rho_{1}}{8\left(\sqrt{1+M_{0}^{2}}+1\right)}, \\
x_{0}=\left(0, r_{0}-\frac{\rho_{1}}{8}\right),
\end{gathered}
$$

we obtain that

$$
B_{r_{0}}\left(x_{0}\right) \subset C_{\frac{\rho_{1}}{8}}^{-}
$$

In the next lemma we continue to use the coordinate system centered in $P$ and the notation described in Definition 1.1 and used above.

We denote

$$
\Gamma_{\frac{\rho_{1}}{M_{0}}, \rho_{1}}^{-}=\left\{\left(x^{\prime}, x_{n}\right) \in \Gamma_{\frac{\rho_{1}}{M_{0}}, \rho_{1}} \mid x_{n}<Z\left(x^{\prime}\right)\right\}
$$

that is

$$
\Gamma_{\frac{\rho_{1}}{M_{0}}, \rho_{1}}^{-}=\Gamma_{\frac{\rho_{1}}{M_{0}}, \rho_{1}} \backslash \bar{\Omega} .
$$


Lemma 6.1 Let $g \in H^{\frac{1}{2}}\left(\Sigma \cap \Gamma_{\frac{\rho_{1}}{M_{0}}, \rho_{1}}\right)$. Then there exists $v \in H^{1}\left(\Gamma_{\frac{\rho_{1}}{M_{0}}, \rho_{1}}^{-}\right)$such that

$$
\left.v\right|_{\Sigma \cap \Gamma_{\frac{\rho}{M_{0}}, \rho_{1}}}=g \text { in the sense of traces, }
$$

and

$$
\|v\|_{H^{1}\left(\Gamma_{\frac{\rho_{1}}{M_{0}, \rho_{1}}}^{-}\right)} \leq C\|g\|_{\left.H^{\frac{1}{2}(\Sigma \cap \Gamma} \frac{\rho_{1}, \rho_{1}}{M_{0}}\right)},
$$

where $C>0$ only depends on $M_{0}$ and $\frac{\rho_{0}}{\rho_{1}}$.

Proof This is a well-known fact. It suffices to prove it first in the reference situation when $Z \equiv 0, M_{0}=1$ and $\rho_{1}=1$, see for instance [57, Lemma 6.9.1].

Next, by a scaling, and by our convention on norms as described in Remark 1.3, we obtain (6.19) when $Z \equiv 0, M_{0}=1$ and $\rho_{1}>0$ is arbitrary. At this stage the constant $C$ in (6.19) shall depend on $\frac{\rho_{0}}{\rho_{1}}$ only.

Finally, by a bilipschitz change of coordinates we may pass to the general (non-flat) case, at the price of admitting that $C$ also depends on $M_{0}$.

Let us now define

$$
\widetilde{u}= \begin{cases}u, & \text { in } \Omega, \\ v, & \text { in } \Gamma_{\frac{\rho_{1}}{M_{0}}, \rho_{1}}^{-},\end{cases}
$$

where $u \in H^{1}(\Omega)$ is a weak solution to the Cauchy Problem 1.6 and $v$ is the function introduced in the previous lemma.

We denote

$$
\Omega_{1}=\Omega \cup\left(\Sigma \cap \Gamma_{\frac{\rho_{1}}{M_{0}}, \rho_{1}}\right) \cup \Gamma_{\frac{\rho_{1}}{M_{0}}, \rho_{1}}^{-} .
$$

We obtain immediately that

$$
\widetilde{u} \in H^{1}\left(\Omega_{1}\right)
$$

and also the following extension theorem.

Theorem 6.2 (Extension) There exist $\tilde{f} \in L^{2}\left(\Omega_{1}\right), \widetilde{F} \in L^{2}\left(\Omega_{1} ; \mathbb{R}^{n}\right)$ such that

$$
\|\widetilde{f}\|_{L^{2}\left(\Omega_{1}\right)}+\frac{1}{\rho_{0}}\|\widetilde{F}\|_{L^{2}\left(\Omega_{1} ; \mathbb{R}^{n}\right)} \leq C \frac{\varepsilon+\eta}{\rho_{0}^{2}}
$$

and $\widetilde{u}$ satisfies in the weak sense

$$
\operatorname{div}(A \nabla \widetilde{u})+c \widetilde{u}=\widetilde{f}+\operatorname{div} \widetilde{F}, \quad \text { in } \Omega_{1} .
$$

Here $C>0$ only depends on $M_{0}, K, \kappa$ and $\frac{\rho_{0}}{\rho_{1}}$. 
Proof Let $\varphi$ be an arbitrary test function in $H_{0}^{1}\left(\Omega_{1}\right)$, with support compactly contained in $\Omega_{1}$. Evidently $\left.\varphi\right|_{\Omega} \in H_{c o}^{1}(\Omega \cup \Sigma)$.

Denoting for simplicity $\Gamma^{-}=\Gamma_{\frac{\rho_{1}}{M_{0}}, \rho_{1}}^{-}$, we compute

$$
\begin{aligned}
-\int_{\Omega_{1}}(A \nabla \widetilde{u} \cdot \nabla \varphi-c \tilde{u} \varphi) & =-\int_{\Omega}(A \nabla u \cdot \nabla \varphi-c u \varphi)-\int_{\Gamma^{-}}(A \nabla v \cdot \nabla \varphi-c v \varphi)= \\
& =-\int_{\Sigma} \psi \varphi+\int_{\Omega}(f \varphi-F \cdot \nabla \varphi)-\int_{\Gamma^{-}}(A \nabla v \cdot \nabla \varphi-c v \varphi) .
\end{aligned}
$$

Let us also denote $\Sigma_{1}=\Sigma \cap \Gamma_{\frac{\rho_{1}}{M_{0}}, \rho_{1}}$ and let us set

$$
\Psi(\varphi)=\int_{\Sigma} \psi \varphi=\rho_{0}^{n-1} \frac{1}{\rho_{0}^{n-1}} \int_{\Sigma} \psi \varphi
$$

We have

$$
|\Psi(\varphi)| \leq \rho_{0}^{n-1}\|\psi\|_{H^{-\frac{1}{2}(\Sigma)}}\left\|\left.\varphi\right|_{\Sigma_{1}}\right\|_{H^{\frac{1}{2}\left(\Sigma_{1}\right)}} \leq C \rho_{0}^{n-2} \eta\|\varphi\|_{H_{0}^{1}\left(\Omega_{1}\right)}
$$

Here $C>0$ is the constant for the trace imbedding $H_{0}^{1}\left(\Omega_{1}\right) \hookrightarrow H^{\frac{1}{2}}\left(\Sigma_{1}\right)$ which only depends on the Lipschitz character of $\Sigma_{1}$. Hence $C$ only depends on $M_{0}$ and $\frac{\rho_{0}}{\rho_{1}}$.

Therefore $\Psi \in H^{-1}\left(\Omega_{1}\right)$ and its norm is bounded as follows

$$
\|\Psi\|_{H^{-1}\left(\Omega_{1}\right)} \leq C \rho_{0}^{n-2} \eta
$$

where $C>0$ is the same constant as above.

By the well-known Riesz representation theorem in Hilbert spaces, we can find $f_{1} \in$ $L^{2}\left(\Omega_{1}\right), F_{1} \in L^{2}\left(\Omega_{1} ; \mathbb{R}^{n}\right)$ such that

$$
\rho_{0}\left\|f_{1}\right\|_{L^{2}\left(\Omega_{1}\right)}+\left\|F_{1}\right\|_{L^{2}\left(\Omega_{1} ; \mathbb{R}^{n}\right)} \leq \frac{\sqrt{2}}{\rho_{0}^{n-1}}\|\Psi\|_{H^{-1}\left(\Omega_{1}\right)} \leq C \frac{\eta}{\rho_{0}}
$$

and

$$
\Psi(\varphi)=\int_{\Omega_{1}} f_{1} \varphi-F_{1} \cdot \nabla \varphi, \quad \text { for every } \varphi \in H_{0}^{1}\left(\Omega_{1}\right)
$$

Note that the powers of $\rho_{0}$ appearing above are calculated according to the fact that the appropriate scalar product for $H^{1}\left(\Omega_{1}\right)$, as derived by our conventions on norms, is given by

$$
\left\langle\varphi, \varphi^{\prime}\right\rangle_{H^{1}\left(\Omega_{1}\right)}=\frac{1}{\rho_{0}^{n}} \int_{\Omega_{1}} \varphi \varphi^{\prime}+\rho_{0}^{2} \nabla \varphi \cdot \nabla \varphi^{\prime}
$$

and, analogously, the $L^{2}(\Sigma)$-scalar product is to be meant as follows

$$
\left\langle\varphi, \varphi^{\prime}\right\rangle_{L^{2}(\Sigma)}=\frac{1}{\rho_{0}^{n-1}} \int_{\Sigma} \varphi \varphi^{\prime}
$$

We define

$$
\widetilde{f}= \begin{cases}f-f_{1}, & \text { in } \Omega \\ c v-f_{1}, & \text { in } \Gamma^{-}\end{cases}
$$




$$
\widetilde{F}= \begin{cases}F-F_{1}, & \text { in } \Omega, \\ A \nabla v-F_{1}, & \text { in } \Gamma^{-} .\end{cases}
$$

We obtain

$$
\begin{gathered}
\|\widetilde{f}\|_{L^{2}\left(\Omega_{1}\right)} \leq\|f\|_{L^{2}(\Omega)}+\left\|f_{1}\right\|_{L^{2}\left(\Omega_{1}\right)}+\frac{\kappa}{\rho_{0}^{2}}\|v\|_{L^{2}\left(\Gamma^{-}\right)}, \\
\|\widetilde{F}\|_{L^{2}\left(\Omega_{1} ; \mathbb{R}^{n}\right)} \leq\|F\|_{L^{2}\left(\Omega ; \mathbb{R}^{n}\right)}+\left\|F_{1}\right\|_{L^{2}\left(\Omega_{1} ; \mathbb{R}^{n}\right)}+K\|\nabla v\|_{L^{2}\left(\Gamma^{-} ; \mathbb{R}^{n}\right)} .
\end{gathered}
$$

Hence

$$
\begin{aligned}
& \|\widetilde{f}\|_{L^{2}\left(\Omega_{1}\right)}+\frac{1}{\rho_{0}}\|\widetilde{F}\|_{L^{2}\left(\Omega_{1} ; \mathbb{R}^{n}\right)} \leq \\
\leq & \left(\|f\|_{L^{2}(\Omega)}+\frac{1}{\rho_{0}}\|F\|_{L^{2}\left(\Omega ; \mathbb{R}^{n}\right)}\right)+\left(\left\|f_{1}\right\|_{L^{2}\left(\Omega_{1}\right)}+\frac{1}{\rho_{0}}\left\|F_{1}\right\|_{L^{2}\left(\Omega_{1} ; \mathbb{R}^{n}\right)}\right)+\frac{C}{\rho_{0}^{2}}\|v\|_{H^{1}\left(\Gamma^{-}\right)},
\end{aligned}
$$

where $C>0$ only depends on $\kappa$ and $K$. Thus, recalling Lemma 6.1 and the assumptions on $f, F, g$ and $\psi$, we deduce (6.23) and from (6.25) we obtain (6.24).

Remark 6.3 It is evident how, by the above theorem, we can translate a Cauchy problem into a problem of propagation of smallness. The philosophy is as follows. Whenever we are given $\rho_{1}>0$ and $P \in \Sigma$ such that $\Gamma_{\frac{\rho_{1}}{M_{0}}, \rho_{1}}(P) \cap \Sigma$ is a Lipschitz graph, and once we have shown that $\left.\widetilde{u}\right|_{\Gamma^{-}}=v$ is "small" in $\Gamma_{\frac{\rho_{1}}{M_{0}}, \rho_{1}}^{-}$, then we can propagate such a "smallness" in a Hölder fashion to any compact subset of $\Gamma_{\frac{\rho_{1}}{M_{0}}, \rho_{1}}$. That is, we obtain that $u=\left.\widetilde{u}\right|_{\Omega}$ is also "small" in a Hölder fashion in a region near $P$ inside $\Omega$. This rough argument is made precise in Theorem 1.7, which we are going to prove here below.

For technical reasons, we found it convenient to make use of the augmented domain $\widetilde{\Omega} \subset \Omega_{1}$ rather than $\Omega_{1}$ itself. The advantage is that when $\Omega$ is assumed to be globally Lipschitz, the global Lipschitz regularity of $\widetilde{\Omega}$ is proven in a more transparent way. This fact will be somewhat helpful later on for the proof of Theorem 1.9 ,

Proof of Theorem 1.7 Let us set $x_{0}$ and $r_{0}$ as in (6.14) and in (6.13), respectively. Let $\bar{h}=\min \left\{2 C_{0} \rho_{0}, \frac{r_{0}}{2}\right\}, C_{0}$ as in the thesis of Theorem 4.1. Notice that, by (6.13), $\bar{h}<\frac{\rho_{1}}{8 M_{0}}$. Denote

$$
\widetilde{G}=G \cup \Gamma \frac{\rho_{1}}{8 M_{0}}, \frac{\rho_{1}}{8} .
$$

Recalling (6.9), it is easily verified that $\widetilde{G} \subset \widetilde{\Omega}$, it is connected and also

$$
\operatorname{dist}(\widetilde{G}, \partial \widetilde{\Omega}) \geq \min \left\{h, \frac{\rho_{1}}{8 M_{0}}\right\}=h .
$$

Moreover, by (6.15),

$$
B_{\frac{r_{0}}{2}}\left(x_{0}\right) \subset \mathcal{A} \cap \Gamma \frac{\rho_{1}}{8 M_{0}}, \frac{\rho_{1}}{8} \subset \widetilde{G} .
$$


Hence we can apply Theorem 5.1 with $u, f, F, \Omega, G$ replaced with $\widetilde{u}, \widetilde{f}, \widetilde{F}, \widetilde{\Omega}, \widetilde{G}$ respectively. By Lemma 6.1 and by (6.20) we recall that

$$
\begin{gathered}
\|\widetilde{u}\|_{L^{2}\left(B_{r_{0}}\left(x_{0}\right)\right)} \leq C \eta, \\
\|\widetilde{u}\|_{L^{2}(\widetilde{\Omega})} \leq C\left(E_{0}+\eta\right),
\end{gathered}
$$

where $C>0$ only depends on $M_{0}$ and $\frac{\rho_{0}}{\rho_{1}}$. Moreover, noticing that $\Omega \supset C_{\rho_{1}}^{+}$, it is immediate to estimate $|\widetilde{\Omega}| \leq C|\Omega|$, with $C>0$ an absolute constant. Thus the thesis follows.

Remark 6.4 Observe that in Theorem 1.7 the assumption $u \in H^{1}(\Omega)$ is not really necessary. This assumption is made just for the sake of simplicity. A more appropriate assumption would be

$$
u \in \bigcap_{\bar{K} \subset \Omega \cup \Sigma} H^{1}(K)
$$

Remark 6.5 As is evident from the proof, the stability of Hölder type could be obtained also on any connected subset $G$ of $\Omega$ having a positive distance $h$ from $\Sigma^{\prime}=\partial \Omega \backslash \sigma$ (rather than from $\partial \Omega$ ). However, unless some additional assumption on the shape and regularity of $\partial \Sigma$ is made, it might not be possible to specify in a precise manner how the constant $C$ and the exponent $\delta$ appearing in (1.35) behave with respect to $h$ as $h \rightarrow 0$. This is in fact an important issue in view of obtaining a global stability bound when no global regularity (Lipschitz) information on $\partial \Omega$ is available. See Section 7 below for further discussion on this issue.

Proof of Theorem 1.9 Similarly to what we did in the previous proof, we apply Theorem 5.3 with $u, f, F, \Omega$ replaced with $\widetilde{u}, \widetilde{f}, \widetilde{F}, \widetilde{\Omega}$, respectively. As before, we consider the ball $B_{r_{0}}\left(x_{0}\right)$ as defined in (6.13), (6.14) and we use the fact that, by Lemma 6.1

$$
\begin{gathered}
\|\widetilde{u}\|_{L^{2}\left(B_{r_{0}}\left(x_{0}\right)\right)} \leq C \eta, \\
\|\widetilde{u}\|_{H^{1}(\widetilde{\Omega})} \leq C(E+\eta),
\end{gathered}
$$

with $C>0$ only depending on $M_{0}$ and $\frac{\rho_{0}}{\rho_{1}}$.

\section{A generalization of the stability results for the Cauchy problem}

Theorem 7.1 (Global stability for the Cauchy problem - generalization) Let $u \in$ $H^{1}(\Omega)$ be a solution to the Cauchy Problem 1.6, where $\Sigma$ satisfies the conditions in Definition 1.4 and Definition 1.5, $f \in L^{2}\left(\mathbb{R}^{n}\right)$ and $F \in L^{2}\left(\mathbb{R}^{n} ; \mathbb{R}^{n}\right)$ satisfy (1.29) and $g \in H^{\frac{1}{2}}(\Sigma)$, $\psi \in H^{-\frac{1}{2}}(\Sigma)$ satisfy (1.25). Let us assume that there exists a family $\left\{G_{h}\right\}, 0<h \leq \bar{h}, \bar{h}$ 
as in Theorem 1.7, of connected open sets $G_{h} \subset \Omega$ satisfying the conditions (1.33), (1.34) and also

$$
\left|\Omega \backslash G_{h}\right| \leq Q \rho_{0}^{n}\left(\frac{h}{\rho_{0}}\right)^{\vartheta}
$$

for given $Q, \vartheta>0$. If, given $E>0, p>2$, we a-priori assume that

$$
\|u\|_{L^{p}(\Omega)} \leq E
$$

then we have

$$
\|u\|_{L^{2}(\Omega)} \leq(E+\varepsilon+\eta) \omega\left(\frac{\varepsilon+\eta}{e(E+\varepsilon+\eta)}\right)
$$

where

$$
\omega(t) \leq \frac{C}{(\log |\log t|)^{S}}, \quad \text { for } 0<t<\frac{1}{e},
$$

where $C>0$ and $S, 0<S<1$, only depend on $K, L, \kappa, M_{0}, \frac{\rho_{0}}{\rho_{1}}, \frac{|\Omega|}{\rho_{0}^{n}}, Q$, $\vartheta$ and $p$.

Remark 7.2 In a completely analogous fashion, a global estimate of propagation of smallness of $\log$-log-type in a general connected open set $\Omega$ could be stated and proved.

Proof of Theorem 7.1 By Hölder inequality, and by our convention on norms (Remark 1.3), we have

$$
\|u\|_{L^{2}(\Omega)} \leq\left(\frac{|\Omega|}{\rho_{0}^{n}}\right)^{\frac{1}{2}-\frac{1}{p}}\|u\|_{L^{p}(\Omega)} \leq C E,
$$

where $C>0$ only depends on $\frac{|\Omega|}{\rho_{0}^{n}}$ and $p$. Hence, by Theorem 1.7 ,

$$
\|u\|_{L^{2}\left(G_{h}\right)} \leq C_{h}(E+\varepsilon+\eta)\left(\frac{\varepsilon+\eta}{E+\varepsilon+\eta}\right)^{\delta_{h}}
$$

where

$$
C_{h}=C_{1}\left(\frac{|\Omega|}{h^{n}}\right)^{\frac{1}{2}} \quad \text { and } \quad \delta_{h} \geq \alpha^{\frac{C_{2}|\Omega|}{h^{n}}},
$$

with $\alpha \in(0,1)$ only depending on $K, L$ and $\kappa, C_{1}$ only depending on $K, L, \kappa, M_{0}$ and $\frac{\rho_{0}}{\rho_{1}}$, and $C_{2}$ only depending on $K$.

We notice that

$$
\begin{aligned}
& \|u\|_{L^{2}\left(G_{h}\right)} \leq C_{h}(E+\varepsilon+\eta)\left(\frac{\varepsilon+\eta}{E+\varepsilon+\eta}\right)^{\delta_{h}}= \\
& \quad=C_{h} e^{\delta_{h}}(E+\varepsilon+\eta)\left(\frac{\varepsilon+\eta}{e(E+\varepsilon+\eta)}\right)^{\delta_{h}} \leq C_{h} e(E+\varepsilon+\eta)\left(\frac{\varepsilon+\eta}{e(E+\varepsilon+\eta)}\right)^{\delta_{h}} .
\end{aligned}
$$


This last step has no substantial importance, it is made only because, at some later stage, it will be convenient to have that the ratio $\frac{\varepsilon+\eta}{e(E+\varepsilon+\eta)}$ is strictly less than $e^{-1}$.

Again using Hölder inequality, we also have

$$
\|u\|_{L^{2}\left(\Omega \backslash G_{h}\right)} \leq\left(\frac{\left|\Omega \backslash G_{h}\right|}{\rho_{0}^{n}}\right)^{\frac{1}{2}-\frac{1}{p}} E \leq E\left(Q\left(\frac{|\Omega|}{\rho_{0}^{n}}\right)^{\frac{\vartheta}{n}}\left(\frac{h^{n}}{|\Omega|}\right)^{\frac{\vartheta}{n}}\right)^{\frac{1}{2}-\frac{1}{p}}
$$

Setting

$$
s=\frac{h^{n}}{|\Omega|}, \quad 0<s \leq s_{0}=\frac{\bar{h}^{n}}{|\Omega|},
$$

we obtain

$$
\|u\|_{L^{2}(\Omega)} \leq C(E+\varepsilon+\eta)\left(s^{-\frac{1}{2}} \tau^{\alpha^{-C_{2} s}}+s^{D}\right), \quad \text { for every } s \in\left(0, s_{0}\right],
$$

where

$$
\begin{gathered}
\tau=\frac{\varepsilon+\eta}{e(E+\varepsilon+\eta)} \in\left(0, e^{-1}\right), \\
D=\frac{\theta}{n}\left(\frac{1}{2}-\frac{1}{p}\right),
\end{gathered}
$$

and $C$ only depends on $K, L, \kappa, M_{0}, \frac{\rho_{0}}{\rho_{1}}, \frac{|\Omega|}{\rho_{0}^{n}}, Q, \vartheta$ and $p$. Note that also $s_{0}$ only depends on $K, L, \kappa, M_{0}, \frac{\rho_{0}}{\rho_{1}}, \frac{|\Omega|}{\rho_{0}^{n}}$. A simple calculation (see for instance [5, Proof of Proposition 3.1]) gives

$$
\inf _{0<s \leq s_{0}}\left(s^{-\frac{1}{2}} \tau^{\alpha^{-C_{2} s}}+s^{D}\right) \leq C(\log |\log \tau|)^{-S}, \quad \text { for every } \tau \in\left(0, e^{-1}\right)
$$

where $C, S>0$ only depend on $K, L, \kappa, M_{0}, \frac{\rho_{0}}{\rho_{1}}, \frac{|\Omega|}{\rho_{0}^{n}}, Q, \vartheta$ and $p$.

\section{References}

[1] V. Adolfsson and L. Escauriaza. $C^{1, \alpha}$ domains and unique continuation at the boundary. Comm. Pure Appl. Math., 50(10):935-969, 1997.

[2] V. Adolfsson, L. Escauriaza, and C. Kenig. Convex domains and unique continuation at the boundary. Rev. Mat. Iberoamericana, 11(3):513-525, 1995.

[3] S. Agmon. Unicité et convexité dans les problèmes différentiels. Séminaire de Mathématiques Supérieures, No. 13 (Été, 1965). Les Presses de l’Université de Montréal, Montreal, Que., 1966.

[4] G. Alessandrini. Stable determination of a crack from boundary measurements. Proc. Roy. Soc. Edinburgh Sect. A, 123(3):497-516, 1993. 
[5] G. Alessandrini, E. Beretta, E. Rosset, and S. Vessella. Optimal stability for inverse elliptic boundary value problems with unknown boundaries. Ann. Scuola Norm. Sup. Pisa Cl. Sci. (4), 29(4):755-806, 2000.

[6] G. Alessandrini and E. Di Benedetto. Determining 2-dimensional cracks in 3dimensional bodies: uniqueness and stability. Indiana Univ. Math. J., 46(1):1-82, 1997.

[7] G. Alessandrini and L. Escauriaza. Null-controllability of one-dimensional parabolic equations. ESAIM Control Optim. Calc. Var., 14(2):284-293, 2008.

[8] G. Alessandrini and R. Magnanini. Elliptic equations in divergence form, geometric critical points of solutions, and Stekloff eigenfunctions. SIAM J. Math. Anal., 25(5):1259-1268, 1994.

[9] G. Alessandrini and V. Nesi. Beltrami operators, non-symmetric elliptic equations and quantitative Jacobian bounds. Ann. Acad. Sci. Fenn. Math., 34(1):47-67, 2009.

[10] G. Alessandrini and L. Rondi. Stable determination of a crack in a planar inhomogeneous conductor. SIAM J. Math. Anal., 30(2):326-340 (electronic), 1998.

[11] G. Alessandrini and E. Rosset. The inverse conductivity problem with one measurement: bounds on the size of the unknown object. SIAM J. Appl. Math., 58(4):1060-1071 (electronic), 1998.

[12] G. Alessandrini, E. Rosset, and J. K. Seo. Optimal size estimates for the inverse conductivity problem with one measurement. Proc. Amer. Math. Soc., 128(1):53-64, 2000 .

[13] F. J. Almgren, Jr. Dirichlet's problem for multiple valued functions and the regularity of mass minimizing integral currents. In Minimal submanifolds and geodesics (Proc. Japan-United States Sem., Tokyo, 1977), pages 1-6. North-Holland, Amsterdam, 1979.

[14] N. Aronszajn. Sur l'unicité du prolongement des solutions des équations aux dérivées partielles elliptiques du second ordre. C. R. Acad. Sci. Paris, 242:723-725, 1956.

[15] N. Aronszajn, A. Krzywicki, and J. Szarski. A unique continuation theorem for exterior differential forms on Riemannian manifolds. Ark. Mat., 4:417-453 (1962), 1962.

[16] E. Beretta and S. Vessella. Stable determination of boundaries from Cauchy data. SIAM J. Math. Anal., 30(1):220-232 (electronic), 1998.

[17] L. Bers, F. John, and M. Schechter. Partial differential equations. Lectures in Applied Mathematics, Vol. III. Interscience Publishers John Wiley \& Sons, Inc. New YorkLondon-Sydney, 1964.

[18] L. Bers and L. Nirenberg. On a representation theorem for linear elliptic systems with discontinuous coefficients and its applications. In Convegno Internazionale sulle Equazioni Lineari alle Derivate Parziali, Trieste, 1954, pages 111-140. Edizioni Cremonese, Roma, 1955.

[19] R. Brummelhuis. Three-spheres theorem for second order elliptic equations. J. Anal. Math., 65:179-206, 1995. 
[20] A. L. Bukhgeim, J. Cheng, and M. Yamamoto. Uniqueness and stability for an inverse problem of determining a part of boundary. In Inverse problems in engineering mechanics (Nagano, 1998), pages 327-336. Elsevier, Oxford, 1998.

[21] A. L. Bukhgeim, J. Cheng, and M. Yamamoto. Stability for an inverse boundary problem of determining a part of a boundary. Inverse Problems, 15(4):1021-1032, 1999.

[22] B. Canuto, E. Rosset, and S. Vessella. Quantitative estimates of unique continuation for parabolic equations and inverse initial-boundary value problems with unknown boundaries. Trans. Amer. Math. Soc., 354(2):491-535 (electronic), 2002.

[23] T. Carleman. Sur les fonctions inverses des fonctions entières d'ordre fini. Ark. fr Mat., Astron. och Fys., 15(10):7 S., 1921.

[24] T. Carleman. Les fonctions quasi analytiques. Leçons professées au Collège de France. Collection de monographies sur la théorie des fonctions. Paris, Gauthier-Villars, 1926.

[25] T. Carleman. Sur les systèmes linéaires aux dérivées partielles du premier ordre à deux variables. C. R. Acad. Sci., Paris, 197:471-474, 1933.

[26] J. Cheng, Y. C. Hon, and M. Yamamoto. Conditional stability estimation for an inverse boundary problem with non-smooth boundary in $\mathbf{R}^{3}$. Trans. Amer. Math. Soc., 353(10):4123-4138 (electronic), 2001.

[27] J. Cheng, Y. C. Hon, and M. Yamamoto. Conditional stability for an inverse Neumann boundary problem. Appl. Anal., 83(1):49-62, 2004.

[28] H. O. Cordes. Über die eindeutige Bestimmtheit der Lösungen elliptischer Differentialgleichungen durch Anfangsvorgaben. Nachr. Akad. Wiss. Göttingen. Math.-Phys. Kl. IIa., 1956:239-258, 1956.

[29] P. L. Duren. Theory of $H^{p}$ spaces. Pure and Applied Mathematics, Vol. 38. Academic Press, New York, 1970.

[30] E. B. Fabes, N. Garofalo, and F.-H. Lin. A partial answer to a conjecture of B. Simon concerning unique continuation. J. Funct. Anal., 88(1):194-210, 1990.

[31] N. Filonov. Second-order elliptic equation of divergence form having a compactly supported solution. J. Math. Sci. (New York), 106(3):3078-3086, 2001.

[32] N. Garofalo and F.-H. Lin. Monotonicity properties of variational integrals, $A_{p}$ weights and unique continuation. Indiana Univ. Math. J., 35(2):245-268, 1986.

[33] N. Garofalo and F.-H. Lin. Unique continuation for elliptic operators: a geometricvariational approach. Comm. Pure Appl. Math., 40(3):347-366, 1987.

[34] D. Gilbarg and N. S. Trudinger. Elliptic partial differential equations of second order, volume 224 of Grundlehren der Mathematischen Wissenschaften. Springer-Verlag, Berlin, second edition, 1983. 
[35] G. M. Goluzin. Geometricheskaya teoriya funktsii kompleksnogo peremennogo. Second edition. Edited by V. I. Smirnov. With a supplement by N. A. Lebedev, G. V. Kuzmina and Ju. E. Alenicyn. Izdat. "Nauka", Moscow, 1966. English translation: Geometric theory of functions of a complex variable, Translations of Mathematical Monographs, 26 American Mathematical Society, Providence, R.I. 1969.

[36] J. Hadamard. Sur les fonctions entières. Bull. Soc. Math. France, 24:94-96, 1896.

[37] J. Hadamard. Sur les problèmes aux derivées partielles et leur signification physique. Bull. Univ. Princeton, 13, 1902.

[38] J. Hadamard. Lectures on Cauchy's problem in linear partial differential equations. New Haven: Yale University Press; London: Humphrey Milford; Oxford: University Press. VIII u. 316 S., 1923.

[39] J. Hadamard. La théorie des équations aux dérivées partielles. Éditions Scientifiques, Peking, 1964.

[40] P. Hartman and A. Wintner. On the local behavior of solutions of non-parabolic partial differential equations. III. Approximations by spherical harmonics. Amer. J. Math., $77: 453-474,1955$.

[41] J. Heinonen, T. Kilpeläinen, and O. Martio. Nonlinear potential theory of degenerate elliptic equations. Oxford Mathematical Monographs. The Clarendon Press Oxford University Press, New York, 1993.

[42] E. Heinz. Über die Eindeutigkeit beim Cauchyschen Anfangswertproblem einer elliptischen Differentialgleichung zweiter Ordnung. Nachr. Akad. Wiss. Göttingen. IIa., 1955:1-12, 1955.

[43] L. Hörmander. Linear partial differential operators. Die Grundlehren der mathematischen Wissenschaften, Bd. 116. Academic Press Inc., Publishers, New York, 1963.

[44] L. Hörmander. Uniqueness theorems for second order elliptic differential equations. Comm. Partial Differential Equations, 8(1):21-64, 1983.

[45] L. Hörmander. The analysis of linear partial differential operators. III \& IV, volumes 274, 275 of Grundlehren der Mathematischen Wissenschaften [Fundamental Principles of Mathematical Sciences]. Springer-Verlag, Berlin, 1985.

[46] T. Hrycak and V. Isakov. Increased stability in the continuation of solutions to the Helmholtz equation. Inverse Problems, 20(3):697-712, 2004.

[47] V. Isakov. Inverse source problems, volume 34 of Mathematical Surveys and Monographs. American Mathematical Society, Providence, RI, 1990.

[48] V. Isakov. Inverse problems for partial differential equations, volume 127 of Applied Mathematical Sciences. Springer, New York, second edition, 2006.

[49] V. Isakov. Increased stability in the Cauchy problem for some elliptic equations. In Instability in models connected with fluid flows. I, volume 6 of Int. Math. Ser. (N. Y.), pages 339-362. Springer, New York, 2008. 
[50] D. Jerison and C. E. Kenig. Unique continuation and absence of positive eigenvalues for Schrödinger operators. Ann. of Math. (2), 121(3):463-494, 1985. With an appendix by E. M. Stein.

[51] D. S. Jerison and C. E. Kenig. Boundary behavior of harmonic functions in nontangentially accessible domains. Adv. in Math., 46(1):80-147, 1982.

[52] F. John. A note on "improper" problems in partial differential equations. Comm. Pure Appl. Math., 8:591-594, 1955.

[53] F. John. Continuous dependence on data for solutions of partial differential equations with a presribed bound. Comm. Pure Appl. Math., 13:551-585, 1960.

[54] R. J. Knops, editor. Symposium on Non-Well-Posed Problems and Logarithmic Convexity. Lecture Notes in Mathematics, Vol. 316. Springer-Verlag, Berlin, 1973.

[55] H. Koch and D. Tataru. Carleman estimates and unique continuation for second-order elliptic equations with nonsmooth coefficients. Comm. Pure Appl. Math., 54(3):339$360,2001$.

[56] J. Korevaar and J. L. H. Meyers. Logarithmic convexity for supremum norms of harmonic functions. Bull. London Math. Soc., 26(4):353-362, 1994.

[57] A. Kufner, O. John, and S. Fučík. Function spaces. Noordhoff International Publishing, Leyden, 1977.

[58] I. Kukavica. Quantitative uniqueness for second-order elliptic operators. Duke Math. J., 91(2):225-240, 1998.

[59] I. Kukavica and K. Nyström. Unique continuation on the boundary for Dini domains. Proc. Amer. Math. Soc., 126(2):441-446, 1998.

[60] E. M. Landis. On some properties of solutions of elliptic equations. Dokl. Akad. Nauk SSSR (N.S.), 107:640-643, 1956.

[61] E. M. Landis. A three-spheres theorem. Dokl. Akad. Nauk SSSR, 148:277-279, 1963.

[62] M. M. Lavrent'ev. On the Cauchy problem for Laplace equation. Izv. Akad. Nauk SSSR. Ser. Mat., 20:819-842, 1956.

[63] M. M. Lavrent'ev. On the inverse problem of potential theory. Dokl. Akad. Nauk SSSR (N.S.), 106:389-390, 1956.

[64] M. M. Lavrent'ev. O nekotorykh nekorrektnykh zadachakh matematicheskoifiziki. Izdat. Sibirsk. Otdel. Akad. Nauk SSSR, Novosibirsk, 1962. English translation: Some improperly posed problems of mathematical physics, Springer tracts in natural philosophy 11. Springer-Verlag, Berlin, 1967.

[65] M. M. Lavrent'ev, V. G. Romanov, and S. P. Šišatskiǔ. Nekorrektnye zadachi matematicheskoi fiziki $i$ analiza. "Nauka", Moscow, 1980. Italian translation: Problemi non ben posti in fisica matematica e analisi, Pubblicazioni dell'Istituto di Analisi Globale e 
Applicazioni 12. IAGA, Firenze, 1983. English translation: Ill-posed problems of mathematical physics and analysis, Translations of Mathematical Monographs 64. American Mathematical Society, Providence, RI, 1986.

[66] O. Lehto and K. I. Virtanen. Quasiconformal mappings in the plane. Springer-Verlag, New York, second edition, 1973.

[67] C.-L. Lin, G. Nakamura, and J.-N. Wang. Quantitative uniqueness for second order elliptic operators with strongly singular coefficients, 2008. Downloadable at http://arxiv.org/abs/0802.1983.

[68] E. Malinnikova. Propagation of smallness for solutions of generalized Cauchy-Riemann systems. Proc. Edinb. Math. Soc. (2), 47(1):191-204, 2004.

[69] N. Mandache. On a counterexample concerning unique continuation for elliptic equations in divergence form. Mat. Fiz. Anal. Geom., 3(3-4):308-331, 1996. Appeared also in: Math. Phys. Anal. Geom., 1(3):273-292, 1998.

[70] V. Maz'ya and T. Shaposhnikova. Jacques Hadamard, a universal mathematician, volume 14 of History of Mathematics. American Mathematical Society, Providence, RI, 1998.

[71] K. Miller. Nonunique continuation for uniformly parabolic and elliptic equations in self-adjoint divergence form with Hölder continuous coefficients. Arch. Rational Mech. Anal., 54:105-117, 1974.

[72] K. Miller. Total total internal reflection. In Partial differential equations and applications, volume 177 of Lecture Notes in Pure and Appl. Math., pages 255-263. Dekker, New York, 1996.

[73] A. Morassi and E. Rosset. Stable determination of cavities in elastic bodies. Inverse Problems, 20(2):453-480, 2004.

[74] A. Morassi and E. Rosset. Uniqueness and stability in determining a rigid inclusion in an elastic body. Mem. Amer. Math. Soc., 200(938), 2009.

[75] A. Morassi, E. Rosset, and S. Vessella. Size estimates for inclusions in an elastic plate by boundary measurements. Indiana Univ. Math. J., 56(5):2325-2384, 2007.

[76] C. Müller. On the behavior of the solutions of the differential equation $\Delta U=F(x, U)$ in the neighborhood of a point. Comm. Pure Appl. Math., 7:505-515, 1954.

[77] N. S. Nadirashvili. Estimation of the solutions of elliptic equations with analytic coefficients which are bounded on some set. Vestnik Moskov. Univ. Ser. I Mat. Mekh., (2):42-46, 1979 .

[78] N. S. Nadirashvili. Uniqueness and stability of continuation from a set to the domain of solution of an elliptic equation. Mat. Zametki, 40(2):218-225, 1986.

[79] F. Nevanlinna and R. Nevanlinna. Über die Eigenschaften analytischer Funktionen in der Umgebung einer singulären Stelle oder Linie. Acta Soc. Sc. Fennicae, 50(5):46 S., 1922 . 
[80] L. Nirenberg. Uniqueness in Cauchy problems for differential equations with constant leading coefficients. Comm. Pure Appl. Math., 10:89-105, 1957.

[81] L. E. Payne. Bounds in the Cauchy problem for the Laplace equation. Arch. Rational Mech. Anal., 5:35-45, 1960.

[82] L. E. Payne. On a priori bounds in the Cauchy problem for elliptic equations. SIAM J. Math. Anal., 1:82-89, 1970.

[83] L. E. Payne. Improperly posed problems in partial differential equations. Society for Industrial and Applied Mathematics, Philadelphia, Pa., 1975.

[84] L. E. Payne and H. F. Weinberger. New bounds for solutions of second order elliptic partial differential equations. Pacific J. Math., 8:551-573, 1958.

[85] A. Petrosyan. Propagation of smallness and the uniqueness of solutions to some elliptic equations in the plane. J. Math. Anal. Appl., 267(2):460-470, 2002.

[86] A. Pliš. On non-uniqueness in Cauchy problem for an elliptic second order differential equation. Bull. Acad. Polon. Sci. Sér. Sci. Math. Astronom. Phys., 11:95-100, 1963.

[87] J. Priwaloff. Sur les fonctions conjuguées. Bull. Soc. Math. France, 44:100-103, 1916.

[88] C. Pucci. Sui problemi di Cauchy non "ben posti". Atti Accad. Naz. Lincei. Rend. Cl. Sci. Fis. Mat. Nat. (8), 18:473-477, 1955.

[89] C. Pucci. Discussione del problema di Cauchy per le equazioni di tipo ellittico. Ann. Mat. Pura Appl. (4), 46:131-153, 1958.

[90] F. Rellich. Darstellung der Eigenwerte von $\Delta u+\lambda u=0$ durch ein Randintegral. Math. Z., 46:635-636, 1940.

[91] L. Rondi. Optimal stability of reconstruction of plane Lipschitz cracks. SIAM J. Math. Anal., 36(4):1282-1292 (electronic), 2005.

[92] S. Stefanesco, C. Schlumberger, and M. Schlumberger. Sur la distribution électrique potentielle autour d'une prise de terre ponctuelle dans un terrain à couches horizontales, homogènes et isotropes. Radium, (7) 1:132-140, 1930.

[93] D. A. Subbarayappa and V. Isakov. On increased stability in the continuation of the Helmholtz equation. Inverse Problems, 23(4):1689-1697, 2007.

[94] T. Takeuchi and M. Yamamoto. Tikhonov regularization by a reproducing kernel Hilbert space for the Cauchy problem for an elliptic equation. SIAM J. Sci. Comput., 31(1):112-142, 2008.

[95] A. N. Tikhonov. On the stability of inverse problems. C. R. (Doklady) Acad. Sci. URSS (N.S.), 39:176-179, 1943.

[96] G. N. Trytten. Pointwise bounds for solutions of the Cauchy problem for elliptic equations. Arch. Rational Mech. Anal., 13:222-244, 1963. 
[97] S. Vessella. A continuous dependence result in the analytic continuation problem. Forum Math., 11(6):695-703, 1999.

[98] S. Vessella. Quantitative continuation from a measurable set of solutions of elliptic equations. Proc. Roy. Soc. Edinburgh Sect. A, 130(4):909-923, 2000.

[99] S. Vessella. Quantitative estimates of unique continuation for parabolic equations, determination of unknown time-varying boundaries and optimal stability estimates. Inverse Problems, 24(2):023001, 81 pages, 2008.

[100] M. S. Zhdanov and G. V. Keller. The geoelectrical methods in geophysical exploration. Methods in geochemistry and geophysics; 31. Elsevier, Amsterdam etc., 1994. 